\title{
CARMENES input catalogue of $M$ dwarfs
}

\section{High-resolution imaging with FastCam ${ }^{\star}$}

\author{
M. Cortés-Contreras ${ }^{1}$, V. J. S. Béjar ${ }^{2}$, J. A. Caballero ${ }^{3,4}$, B. Gauza $^{2}$, D. Montes ${ }^{1}$, \\ F. J. Alonso-Floriano ${ }^{1}$, S. V. Jeffers ${ }^{5}$, J. C. Morales ${ }^{6}$, A. Reiners ${ }^{5}$, I. Ribas ${ }^{6}$, P. Schöfer ${ }^{5}$, A. Quirrenbach ${ }^{4}$, \\ P. J. Amado ${ }^{7}$, R. Mundt ${ }^{8}$, and W. Seifert ${ }^{4}$
}

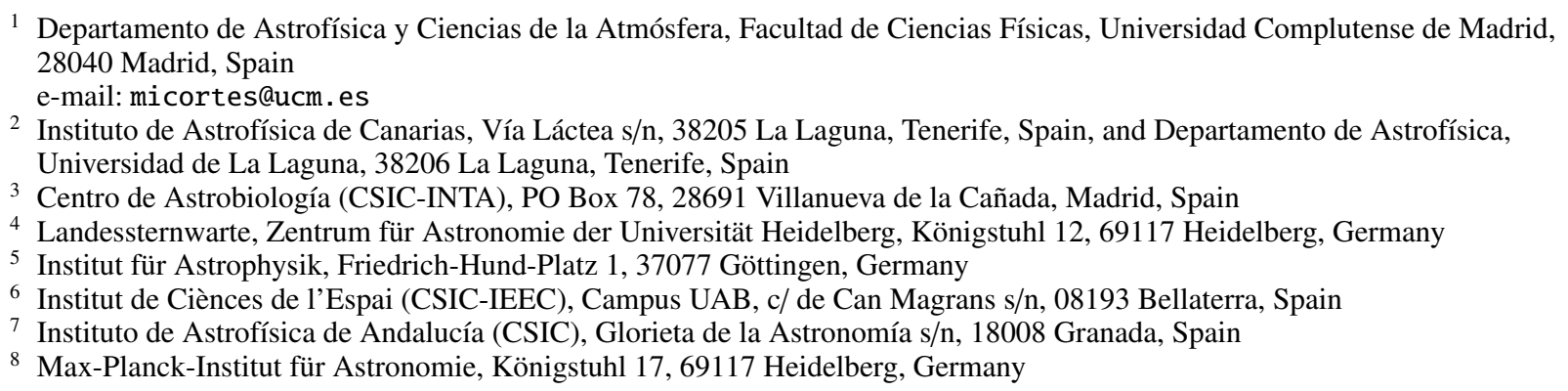

Received 3 June 2016 / Accepted 23 August 2016

\begin{abstract}
Aims. We search for low-mass companions of M dwarfs and characterize their multiplicity fraction with the purpose of helping in the selection of the most appropriate targets for the CARMENES exoplanet survey.

Methods. We obtained high-resolution images in the $I$ band with the lucky imaging instrument FastCam at the $1.5 \mathrm{~m}$ Telescopio Carlos Sánchez for 490 mid- to late-M dwarfs. For all the detected binaries, we measured angular separations, position angles, and magnitude differences in the $I$ band. We also calculated the masses of each individual component and estimated orbital periods, using the available magnitude and colour relations for $\mathrm{M}$ dwarfs and our own $M_{J}$-spectral type and mass- $M_{I}$ relations. To avoid biases in our sample selection, we built a volume-limited sample of M0.0-M5.0 dwarfs that is complete up to 86\% within $14 \mathrm{pc}$.

Results. From the 490 observed stars, we detected 80 companions in 76 systems, of which 30 are new discoveries. Another six companion candidates require additional astrometry to confirm physical binding. The multiplicity fraction in our observed sample is $16.7 \pm 2.0 \%$. The bias-corrected multiplicity fraction in our volume-limited sample is $19.5 \pm 2.3 \%$ for angular separations of 0.2 to 5.0 arcsec $(1.4-65.6 \mathrm{au})$, with a peak in the distribution of the projected physical separations at $2.5-7.5 \mathrm{au}$. For M0.0-M3.5 V primaries, our search is sensitive to mass ratios higher than 0.3 and there is a higher density of pairs with mass ratios over 0.8 compared to those at lower mass ratios. Binaries with projected physical separations shorter than 50 au also tend to be of equal mass. For 26 of our systems, we estimated orbital periods shorter than 50 a, 10 of which are presented here for the first time. We measured variations in angular separation and position angle that are due to orbital motions in 17 of these systems. The contribution of binaries and multiples with angular separations shorter than $0.2 \mathrm{arcsec}$, longer than $5.0 \mathrm{arcsec}$, and of spectroscopic binaries identified from previous searches, although not complete, may increase the multiplicity fraction of $\mathrm{M}$ dwarfs in our volume-limited sample to at least $36 \%$.
\end{abstract}

Key words. binaries : close - stars: late-type - stars: low-mass

\section{Introduction}

The multiplicity of low-mass stars provides constraints to models of stellar and planet formation and evolution (Goodwin et al. 2007; Burgasser et al. 2007; Duchêne \& Kraus 2013). M dwarfs, which have approximate masses of between 0.1 and $0.6 M_{\odot}$, account for two thirds of the stars in the solar neighbourhood and probably the Galaxy. However, in spite of their abundance and the increasing number of M-dwarf high-resolution imaging surveys in the past decade (Beuzit et al. 2004; Law et al. 2008; Bergfors et al. 2010; Janson et al. 2012, 2014a; Jódar et al. 2013; Bowler et al. 2015; Ward-Duong et al. 2015), the multiplicity

\footnotetext{
* Tables A.1-A.6 are only available at the CDS via anonymous ftp to cdsarc.u-strasbg. fr (130.79.128.5) or via http://cdsarc.u-strasbg.fr/viz-bin/qcat?]/A+A/597/A47
}

of M dwarfs is not yet well constrained, at least by comparison with the better determination for Sun-like stars (Duquennoy \& Mayor 1991; Raghavan et al. 2010; Tokovinin 2011). Published values range between $13.6 \%$ and $42 \%$. Thus, the binary fraction of M dwarfs seems intermediate between the one of Sun-like stars and very low mass binaries. In Table 1 we summarise the multiplicity fractions and semi-major axis coverage of some of the main multiplicity surveys carried out from F6 to $\mathrm{T}$ dwarfs.

The typical separation of low-mass stars in a binary system tends to decrease with the mass of the primary, which makes the detection of faint companions at resolvable separations more difficult (Jeffries \& Maxted 2005; Burgasser et al. 2007; Caballero 2007; Bate 2012; Luhman 2012). In addition, the presence of a stellar companion influences planet formation (Wang et al. 2014a,b, 2015a,b). The limited number of exoplanet 
Table 1. Stellar multiplicity fractions.

\begin{tabular}{|c|c|c|c|c|c|}
\hline Reference & $\begin{array}{l}\text { Investigated } \\
\text { spectral type }\end{array}$ & $\begin{array}{l}d_{\lim } \\
{[\mathrm{pc}]}\end{array}$ & $\begin{array}{l}\text { Multiplicity } \\
\text { fraction [\%] }\end{array}$ & $\begin{array}{l}\text { Projected physical } \\
\text { separation, } s \text { [au] }\end{array}$ & $\begin{array}{l}\text { Survey } \\
\text { method }^{a}\end{array}$ \\
\hline Duquennoy \& Mayor (1991) & F7-G9 & 22 & $\sim 65$ & $\sim 0.01-225$ & RV, WI \\
\hline Raghavan et al. (2010) & $\sim \mathrm{F} 6-\mathrm{K} 3$ & 25 & $44 \pm 3$ & $\sim 0.005-100000$ & $\mathrm{RV}, \mathrm{AO}, \mathrm{S}, \mathrm{WI}$ \\
\hline Reid \& Gizis (1997) & K2-M6 & 8 & 32 & $\sim 0.1-1800$ & RV, S, WI \\
\hline Leinert et al. (1997) & M0-M6 & 5 & $26 \pm 9$ & $\sim 1-100$ & $\mathrm{~S}$ \\
\hline Fischer \& Marcy (1992) & M & 20 & $42 \pm 9$ & $0-10000$ & RV, WI \\
\hline Jódar et al. (2013) & K5-M4 & 25 & $20.3_{-5.2}^{+6.9}$ & $\sim 0-80$ & LI \\
\hline Ward-Duong et al. (2015) & K7-M6 & 15 & $23.5 \pm 3.2$ & $\sim 3-10000$ & $\mathrm{AO}, \mathrm{WI}$ \\
\hline Bergfors et al. (2010) & M0.0-M6.0 & 52 & $32 \pm 6$ & $3-180$ & LI \\
\hline Janson et al. (2012) & M0.0-M5.0 & 52 & $27 \pm 3$ & $3-227$ & LI \\
\hline Law et al. (2008) & M4.5-M6.0 & $\langle 15.4\rangle$ & $13.6_{-4.0}^{+6.5}$ & $\sim 0-80$ & LI \\
\hline Siegler et al. (2005) & M6.0-M7.5 & 30 & $9_{-3}^{+4}$ & $\geqslant 3$ & $\mathrm{AO}$ \\
\hline Janson et al. (2014a) & M5.0-M8.0 & 36 & $21-27$ & $\sim 0.5-100$ & LI \\
\hline Close et al. (2003) & M8.0-L0.5 & 33 & $15 \pm 7$ & $<15$ & $\mathrm{AO}$ \\
\hline Bouy et al. (2003) & M7.0-L8.0 & 20 & $10-15$ & $1-8$ & HST \\
\hline Reid et al. (2008) & $\mathrm{L}$ & 20 & $12.5_{-3.0}^{+5.3}$ & $<3$ & HST \\
\hline Burgasser et al. (2003) & $\mathrm{T}$ & $\langle 10\rangle$ & $9_{-4}^{+15}$ & $1-5$ & HST \\
\hline
\end{tabular}

Notes. ${ }^{(a)}$ AO: Adaptive optics; HST: Hubble Space Telescope; LI: Lucky imaging; RV: Radial velocity; S: Speckle; WI: Wide-field imaging.

hosts in binary and multiple systems (Mugrauer et al. 2007; Mugrauer \& Neuhäuser 2009; Ginski et al. 2015) and the relatively small number of $\mathrm{M}$ dwarfs with known exoplanets detected with radial-velocity and transit methods (Rivera et al. 2005; Charbonneau et al. 2009; Bonfils et al. 2013) prevents a significant statistical analysis of how stellar multiplicity at such low masses affects planet formation.

Because of their low effective temperatures, $M$ dwarfs emit the bulk of their energy in the near-infrared. It makes them difficult to observe with the required radial-velocity precision with the current spectrographs for exoplanet hunting (e.g. HARPS at the $3.6 \mathrm{~m}$ ESO La Silla Telescope, HARPS-N at the $3.6 \mathrm{~m}$ TNG, and UVES at the $8.2 \mathrm{~m}$ ESO VLT), which operate in the optical. The prompt development of stable near-infrared spectrographs with wide wavelength coverage and high spectral resolution for radial-velocity surveys of $\mathrm{M}$ dwarfs has therefore been identified as critical by numerous decadal panels, funding agencies, and international consortia. Some noteworthy high-resolution near-infrared spectrographs currently under developement are IRD at $8.2 \mathrm{~m}$ Subaru (Tamura et al. 2012), HPF at 9.2 m HET (Mahadevan et al. 2014), and SPIRou at $3.6 \mathrm{~m}$ CFHT (Donati et al. 2014). The high-resolution spectrograph CARMENES (Amado et al. 2013; Quirrenbach et al. 2014 ${ }^{1}$ ) at $3.5 \mathrm{~m}$ Calar Alto covers from $520 \mathrm{~nm}$ to $1710 \mathrm{~nm}$ and has started its science survey in January 2016.

CARMENES is the name of the double-channel spectrograph (near-infrared and optical) of the Spanish-German consortium that built it, and of the science project that is being carried out during guaranteed-time observations (GTO). For at least 600 GTO clear nights in the time frame between 2016 and 2018, CARMENES will spectroscopically monitor about 300 carefully selected M dwarfs with the goal of detecting low-mass planets in their habitable zones. With a long-term $1 \mathrm{~m} \mathrm{~s}^{-1}$ radialvelocity precision, the consortium aims at being able to detect

\footnotetext{
1 http://carmenes.caha.es
}

$2 M_{\oplus}$ planets orbiting in the habitable zone of M5 V stars and super-Earths around earlier stars (García-Piquer et al. 2016). In addition to the detection of the individual planets themselves, the ensemble of objects will provide sufficient statistics to assess the overall distribution of planets around M dwarfs: frequency, masses, and orbital parameters.

To optimise the observational strategy of the instrument and its scientific return, the consortium has built Carmencita, the CARMENES input catalogue (Caballero et al. 2013; Quirrenbach et al. 2015; Alonso-Floriano et al. 2015). It consists of almost 2200 of the brightest M dwarfs of each spectral subtype observable from Calar Alto, from which we will select the approximately 300 single GTO stars. By single we mean stars without close visual (physically bound) or optical (unbound) stellar or substellar companions that may induce real or artificial radial-velocity variations and, therefore, contaminate the precise CARMENES measurements (Guenther \& Wuchterl 2003; Ehrenreich et al. 2010; Guenther \& Tal-Or 2010; Bonfils et al. 2013).

As part of our efforts to determine the multiplicity of $\mathrm{M}$ dwarfs and to select the best targets for radial-velocity surveys for exoplanets, we performed a high-resolution imaging search of close companions with the lucky imaging instrument FastCam at the Telescopio Carlos Sánchez, as described in this paper. Preliminary results of this work were presented as conference proceedings by Béjar et al. (2012) and Cortés-Contreras et al. (2015a,b). This paper is the second item of the series called the CARMENES input catalogue of M dwarfs. In the first paper, Alonso-Floriano et al. (2015) carried out a low-resolution optical spectroscopic analysis of a number of poorly known dwarfs to constrain their spectral types. Furthermore, this work will soon be complemented with on-going searches of unresolved spectroscopic binaries and triples identified in a large collection of high-resolution optical spectra (Montes et al. 2015; Jeffers et al., in prep.) and of wide companions to $M$ dwarfs supported by 


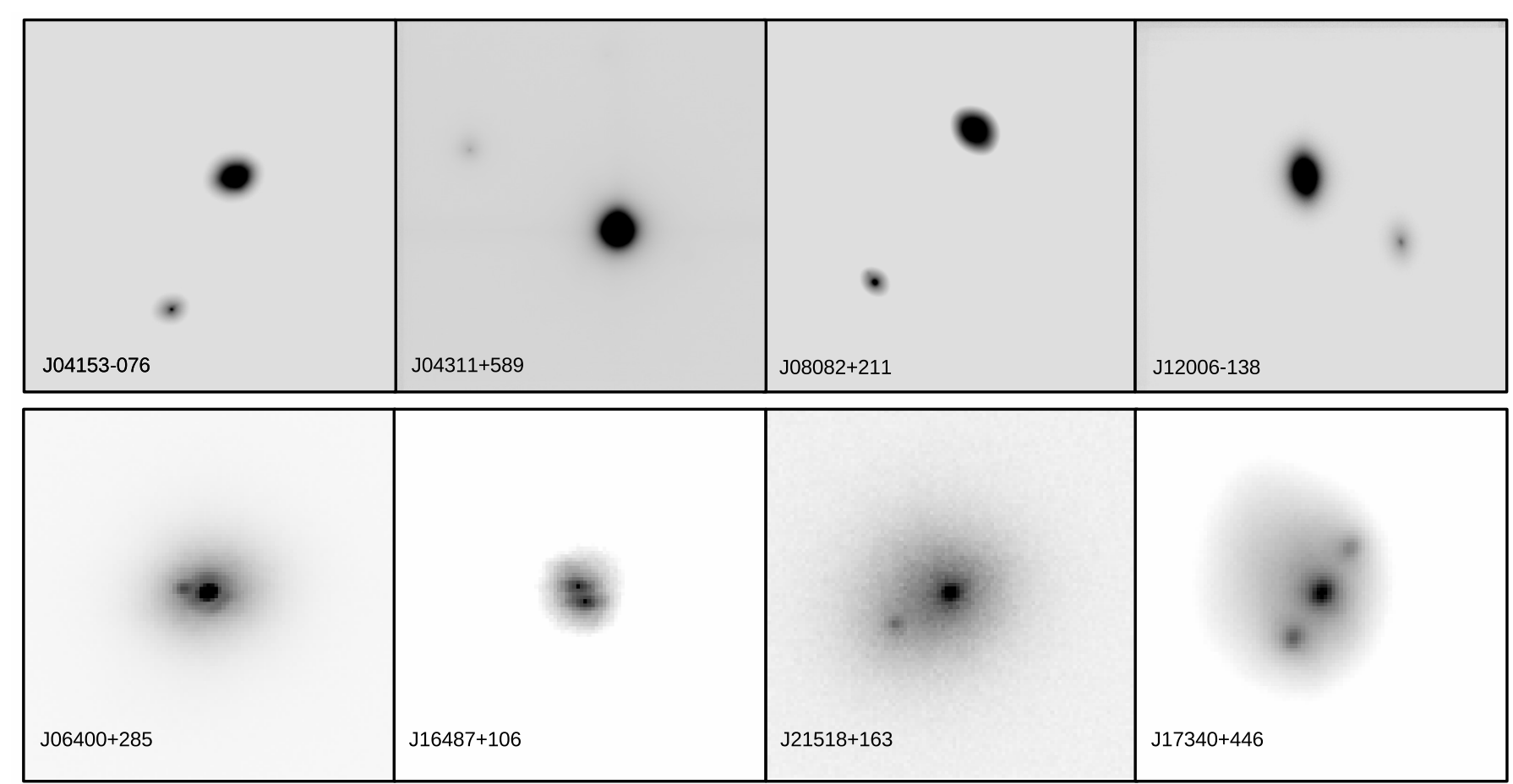

Fig. 1. Selection of images of multiple systems identified by us with FastCam. North is up and east is left. The upper row scale is $20 \times 20$ arcsec ${ }^{2}$, that of the lower row $4 \times 4 \operatorname{arcsec}^{2}$. Images at the top were obtained with the shift \& add mode, while the bottom images were obtained with the lucky image mode. The bottom right image $(\mathrm{J} 17340+446)$ is an examlpe of the so-called false triple effect.

virtual observatory tools (cf., Cortés-Contreras et al. 2013, 2014; Alonso-Floriano et al. 2015).

\section{Observations}

Of the almost $2200 \mathrm{M}$ dwarfs currently in Carmencita, we selected 490 Carmencita targets for being observed with the FastCam lucky imager (Oscoz et al. 2008) at the $1.5 \mathrm{~m}$ Telescopio Carlos Sánchez at the Observatorio del Teide (Tenerife, Spain). The high-resolution imager FastCam is equipped with an L3CCD Andor $512 \times 512$ detector with very low electron noise and high readout speed. It has a field of view of $21.2 \times 21.2 \operatorname{arcsec}^{2}$ and an approximate pixel size and orientation of the detector of 0.0425 arcsec and $91.9 \mathrm{deg}$, respectively. FastCam delivers nearly diffraction-limited images, which at the Telescopio Carlos Sánchez and in the $I$ band have full-width at half maxima of approximately 0.15 arcsec.

We carried out the observations during 26 nights in 15 runs from October 2011 to January 2016. For each target, we obtained typically ten blocks of 1000 frames each in the Johnson-Cousins I band using the electron multiplication mode. Typical frame exposure times were in the $35-50 \mathrm{~ms}$ range. On average, each star was imaged during $500 \mathrm{~s}$ in total. The typical Strehl ratio in our observations varies with the percentage of the best-quality frames chosen in the reduction process: from 0.2 for the $100 \%$ to 0.4 for the $1 \%$. For astrometric calibration purposes, we also observed the globular cluster M3 and 18 astrometric standard binary stars from the Aitken Double Star catalogue (ADS - Aitken 1932; Scardia et al. 1995) with the same method and on several occasions.

Each frame was bias subtracted and then processed with the FastCam dedicated software developed at the Universidad Politécnica de Cartagena (see Labadie et al. 2010; Jódar et al. 2013). We ran the lucky image (on five blocks) and shift \& add processing modes separately. The first allows selecting the fraction of the best-quality frames (we chose $1 \%, 10 \%$, and $50 \%$ ), aligns the selected frames using the brightest speckle, and combines them, producing six final lucky images per target. The second mode aligns all the block frames and then combines them, resulting in one unique image per target. Shift \& add produces deeper images than the lucky image mode, but with slightly poorer resolution. The M3 standard field was reduced only with the shift $\&$ add mode. In Fig. 1 we show a selection of the processed images at two different spatial scales.

In Table A.1, we provide the list of 490 observed M-dwarf targets with the following column information: identification number, our Carmencita identifier (Quirrenbach et al. 2015; Alonso-Floriano et al. 2015), J2000 coordinates and J-band magnitude from the Two-Micron All-Sky Survey (Skrutskie et al. 2006), spectral type and its reference, distance and its reference, and the FastCam observation date and exposure time. Figure 2 shows the histograms of spectral types, $J$-band magnitudes, heliocentric distances, and total proper motions of the observed sample. Spectral types range from M0.0 V to M7.0 V, $J$ from $4.2 \mathrm{mag}$ to $10.4 \mathrm{mag}$, distances from $1.8 \mathrm{pc}$ to $39.1 \mathrm{pc}$, and proper motions from $0.03 \operatorname{arcsec} \mathrm{a}^{-1}$ to $10.6 \operatorname{arcsec}^{-1}$. Because of their closeness, $97 \%$ of our targets have total proper motions larger than 100 mas a $^{-1}$.

Our sample of 490 observed Carmencita targets consisted mainly of the brightest stars in the $J$ band for each spectral subtype (see Sect. 2 in Alonso-Floriano et al. 2015) that were (i) not known spectroscopic binaries; (ii) not resolved systems with visual or optical companions at angular separations smaller than 5 arcsec; and (iii) not studied with high-resolution imaging devices before the start of our observations by speckle, adaptive optics, or lucky imaging (Beuzit et al. 2004; Law et al. 2008; Bergfors et al. 2010; Jódar et al. 2013; Janson et al. 2012). Some high-resolution imaging (Janson et al. 2014a; Ansdell et al. 2015; Bowler et al. 2015; Ward-Duong et al. 2015) and spectroscopic (Bonfils et al. 2013; Llamas 2014; Schöfer 2015) 

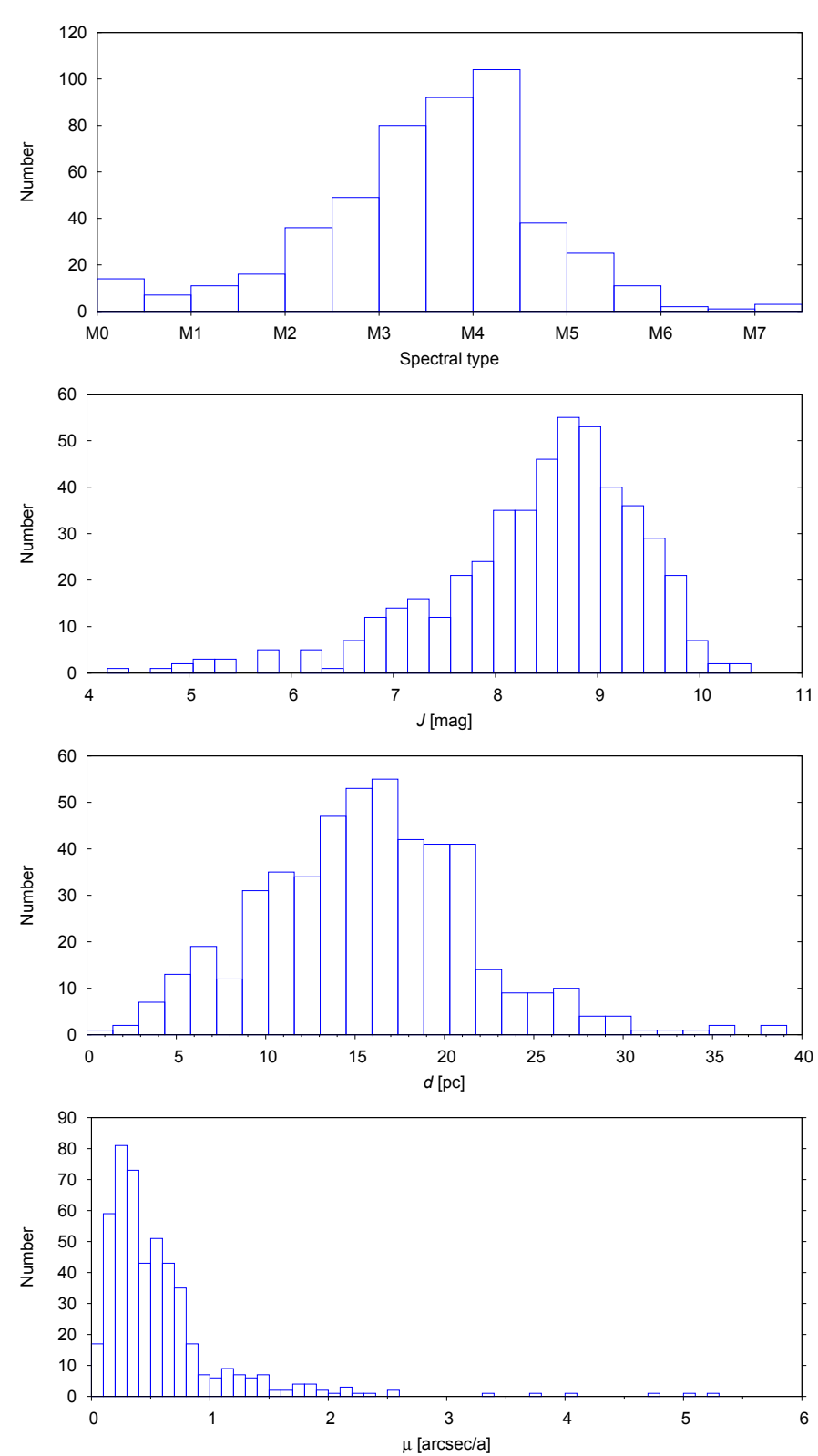

Fig. 2. Distributions of spectral type, $J$-band magnitude, distance, and proper motion of the 490 observed M-dwarf targets. The sizes of the bins follow the definitions given by Freedman \& Diaconis (1981). The lowest panel does not display Barnard's star, with $\mu=10.4 \operatorname{arcsec~}^{-1}$.

surveys have been performed afterwards and have tabulated several objects in common with our target list. In addition, we also observed (a) some dubious or poorly investigated close multiple systems (including spectroscopic binary candidates); (b) a few stars with possible visual companions at angular separations smaller than 5 arcsec that needed confirmation or better characterisation; and (c) four known binaries with estimated orbital periods shorter than five years that were previously proposed for follow-up by Cortés-Contreras et al. (2013): J05085-181 (GJ 190), J13317+292 (DG CVn), J23174+196 (G 067-053), and J23455-161 (LP 823-004).

To confirm the physical binding of pairs (i.e. that the components share a common proper motion), we observed 54 targets more than once, and up to eight times. Accounting for the $490 \mathrm{M}$ dwarfs, 18 ADS pairs and M3 calibration field, and
Table 2. FastCam adopted plate scale and orientation for each run night.

\begin{tabular}{|c|c|c|c|c|}
\hline \multirow{2}{*}{$\begin{array}{l}\text { Observation } \\
\text { date }^{a}\end{array}$} & \multicolumn{2}{|c|}{ Pixel scale [mas/pix] } & \multicolumn{2}{|c|}{ Orientation [deg] } \\
\hline & $x$ & $y$ & $x$ & $y$ \\
\hline 23 Oct. $2011^{*}$ & 42.25 & 42.56 & 92.08 & 91.60 \\
\hline 24 Oct. 2011 & 42.25 & 42.56 & 92.08 & 91.60 \\
\hline 25 Oct. 2011 & 42.25 & 42.56 & 92.08 & 91.60 \\
\hline 30 Jan. 2012 & 42.25 & 42.56 & 92.08 & 91.60 \\
\hline 31 Jan. 2012 & 42.25 & 42.56 & 92.08 & 91.60 \\
\hline 25 Mar. 2012* & 42.31 & 42.61 & 91.79 & 91.64 \\
\hline 26 Mar. 2012* & 42.30 & 42.62 & 91.82 & 91.65 \\
\hline 27 Mar. 2012 & 42.30 & 42.62 & 91.82 & 91.65 \\
\hline 10 Jul. 2012* & 42.48 & 42.61 & 92.11 & 91.91 \\
\hline 11 Jul. $2012 *$ & 42.49 & 42.64 & 92.03 & 91.77 \\
\hline 12 Jul. $2012 *$ & 42.32 & 42.54 & 91.96 & 91.99 \\
\hline 16 Sep. 2012 & 42.32 & 42.54 & 91.96 & 91.99 \\
\hline 17 Sep. 2012 & 42.32 & 42.54 & 91.96 & 91.99 \\
\hline 13 Jan. $2013^{*}$ & 42.26 & 42.69 & 91.94 & 91.63 \\
\hline 14 Jan. 2013* & 42.21 & 42.59 & 91.85 & 91.63 \\
\hline 28 Feb. 2014* & 42.26 & 42.69 & 91.99 & 91.70 \\
\hline 01 Mar. 2014 & 42.26 & 42.69 & 91.99 & 91.70 \\
\hline 02 Mar. 2014 & 42.26 & 42.69 & 91.99 & 91.70 \\
\hline 22 May 2014 & 42.26 & 42.69 & 91.99 & 91.70 \\
\hline 09 Dec. $2014 *$ & 42.26 & 42.99 & 91.97 & 91.96 \\
\hline 14 Apr. $2015^{*}$ & 42.28 & 42.37 & 92.18 & 91.65 \\
\hline 15 Apr. 2015 & 42.28 & 42.37 & 92.18 & 91.65 \\
\hline 09 Jun. 2015 & 42.28 & 42.37 & 92.18 & 91.65 \\
\hline 29 Jul. 2015 & 42.28 & 42.37 & 92.18 & 91.65 \\
\hline 17 Nov. 2015 & 42.28 & 42.37 & 92.18 & 91.65 \\
\hline 07 Jan. 2016 & 42.28 & 42.37 & 92.18 & 91.65 \\
\hline
\end{tabular}

Notes. ${ }^{(a)}$ M3 calibration field was observed on nights marked with an asterisk.

the different epochs, we acquired 7670 images in total with FastCam.

\section{Analysis}

\subsection{Astrometry}

The first step of the analysis was computing the pixel size and detector orientation with common IRAF tasks (Tody 1986). To do this, we determined the centroids of the brightest stars in the M3 standard field with imcentroid. Using the celestial coordinates in the ACS Survey of Galactic Globular Clusters (Sarajedini et al. 2007) and the pixel coordinates in our images, we then determined the transformation equations with ccmap by fitting to a general transformation of order two. Table 2 lists the pixel scales and orientations of the detector for each night. For nights without M3 images, we used the calibration of the closest night with computed plate solution. Pixel scale and rotation angle in the centre of the detector in the $x$ and $y$ axes are similar within the different campaigns with almost negligible variations from night to night. Their mean values are $42.31 \pm 0.09 \mathrm{mas} / \mathrm{pixel}$ and $42.63 \pm 0.15 \mathrm{mas} /$ pixel in pixel scale and $91.98 \pm 0.12 \mathrm{deg}$ and $91.74 \pm 0.15 \mathrm{deg}$ in orientations of the detector in the $x$ and $y$ axes, respectively. The uncertainties are the standard deviations of the measurements. 
To double-check that our astrometric solutions were correct, we calculated angular separations $(\rho)$ and position angles $(\theta)$ for each ADS binary. To do that, we measured the $x$ and $y$ positions of each star with imcentroid, and transformed them into equatorial coordinates using the astrometric solution of the corresponding night with cctran. Table A. 2 shows the previously published values of $\rho, \theta$, the epochs of observation and references, and our measured values in different epochs. Our errors in $\rho$ and $\theta$ were derived from the standard deviation of the measurements in all images within the same night and the determined astrometric solutions on different nights. In general, the measured values of $\rho$ and $\theta$ of the same pair on different nights were consistent within $3 \sigma$ between them and with tabulated values from recent works. In some cases, the quality of our measurements surpassed previous publications.

We carried out a visual inspection for companions to our 490 Carmencita targets and found 137 additional sources in 116 systems, for which we measured the relative positions and position angles following the same procedure as described above for the ADS binaries. In some epochs of nine stars with companions very close to the resolution limit of our images, we were unable to measure the photocentroid of both components with imcentroid and, hence, we used the brightest pixel to measure their positions. In these cases, the uncertainties in the determination of $\rho$ and $\theta$ were larger and we adopted a typical error bar of one pixel.

We classified the 137 sources into three groups: (i) 51 optical companions (i.e. unbound, Table A.3); (ii) 80 physical companions (i.e. bound, Table A.4); and (iii) six unconfirmed companions (bottom of Table A.4). For the classification, we used old photographic plate digitisations and all-sky surveys provided by the Aladin sky atlas (Bonnarel et al. 2000), previous astrometry tabulated by the Washington Double Star Catalogue (WDS, Mason et al. 2001) and/or our own multi-epoch astrometric measurements together with the target proper motions (mostly from van Leeuwen 2007 and Röser et al. 2010).

Most of the 51 optical companions are null-proper-motion point-like sources in photographic plates of the first National Geographic Society - Palomar Observatory Sky Survey in the mid-1950s. For the rest of the companions, we performed a multi-epoch analysis of their relative positions. We considered as optical (unbound) companions those that show $\rho$ and $\theta$ values in different epochs consistent within $3 \sigma$ with null proper motion and inconsistent by more than $3 \sigma$ with the proper motion of the M dwarfs. Otherwise, we considered them as physically bound. For five of the six unconfirmed binaries, we only had one epoch, and for the other $(\mathrm{J} 07349+147)$, the $\rho$ and $\theta$ values at different epochs did not allow us to distinguish between null or common proper motion.

Figure 3 displays the measured $\rho$ and $\theta$ values of all the detected pairs. It shows a homogeneous distribution of the position angle of the companions, which discards possible false detections associated with, for example, optical ghosts.

\subsection{Photometry}

In Table A.4 we list magnitude differences in the $I$ band for the 80 physical and six likely physical pairs. To measure the magnitude difference of the binaries, we performed aperture and point spread function (PSF) photometry using the phot, psf, and allstar routines in the daophot package of IRAF.

For wide enough pairs, we used the primary star PSF as a reference for the secondary. In these cases, magnitude differences from aperture photometry and PSF fitting did not differ

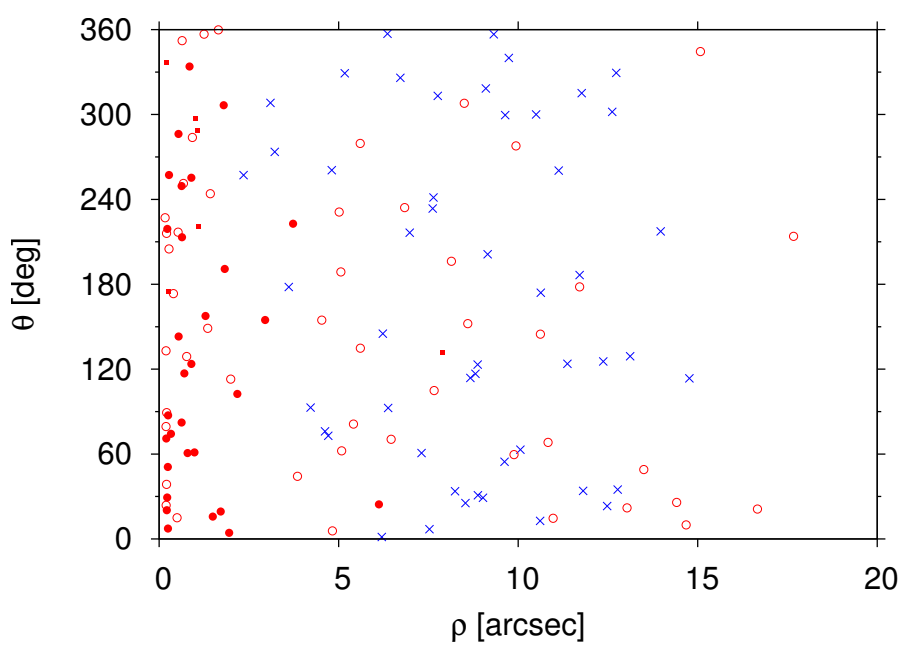

Fig. 3. Diagram of $\theta$ vs. $\rho$ for all the 137 measured pairs. Filled red circles are new physically bound pairs, small filled red squares are unconfirmed related pairs, open red circles are known physically bound pairs, and blue crosses are optically unrelated pairs.

significantly. Since the PSF varies depending on the focus and sky position, for close pairs we chose the most appropriate single star observed during the same night as a reference to compute the PSF. For five pairs, we were unable to measure the $\Delta I$ between components using PSF photometry, and we estimated it from the peak flux ratio of the PSF subtracted image, and for J23455-161, we perceived the companion and could not measure the magnitude difference.

A few close pairs showed a so-called false triple effect associated with the reduction process by the FastCam software, based on the selection of the brightest pixel. When both components are of similar brightness, this software may not distinguish between the primary and secondary and, in the process of aligning, selects the brightest pixel in one or another star, resulting in an apparent triple system. For equal brightness binaries, this may lead to a degeneracy in the determination of the position angle of $180 \mathrm{deg}$. The option 2 stars in the FastCam reduction software, which takes this ambiguity into account, solved this effect in most cases. For the rest, we determined the real flux ratio of the pair by following the procedure described by Law (2006):

$F_{R}=\frac{2 I_{13}}{I_{12} I_{13}+\sqrt{I_{12}^{2} I_{13}^{2}-4 I_{12} I_{13}}}$,

where $I_{12}=F_{1} / F_{2}$ and $I_{13}=F_{1} / F_{3}$, and $F_{1}, F_{2}$ and $F_{3}$ are the fluxes of the images in the positions of the true primary, true secondary, and spurious tertiary, respectively.

In Fig. 4 we plot the measured magnitude differences in the $I$ band and angular separations of the companions. Most of them are of similar brightness $(\Delta I \approx 0.0-1.0 \mathrm{mag})$ and are located at angular separations smaller than 2.5 arcsec. Figure 4 also shows the contrast curves of our survey as a function of angular separation. The maximum magnitude difference in each stacked image depends on the brightness of the primary star. For this reason, we considered three different groups in our sample according to their $I$ magnitude, from which we selected four single stars covering different spectral types to obtain a representative mean contrast curve. For each star, we estimated the detection limit as a function of the angular separation as three times the standard deviation of the number of counts in ten-pixel-wide annuli centred on the target. This detection limit was converted into 


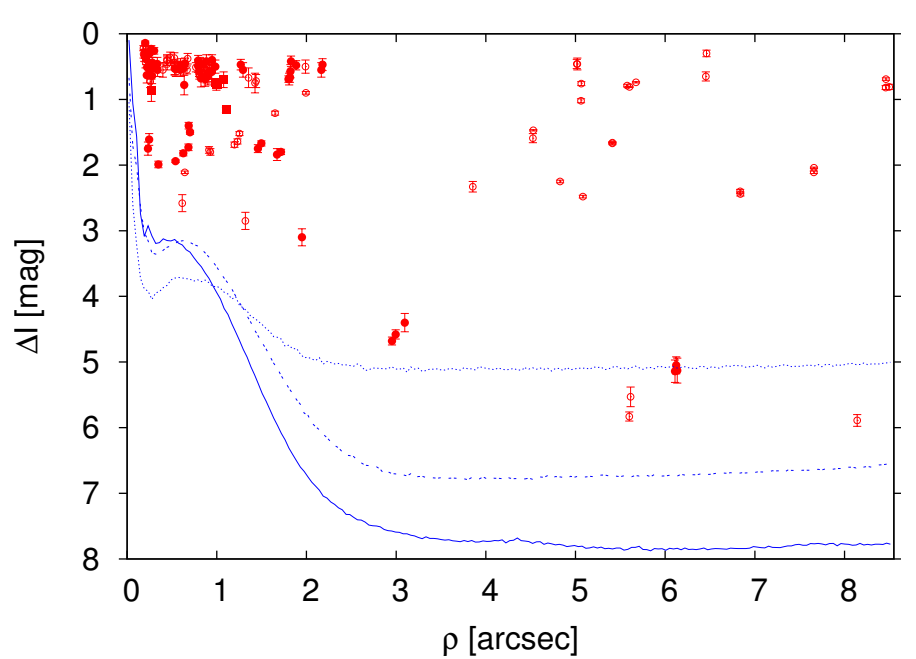

Fig. 4. Diagram of $\Delta I$ vs. $\rho$ up to 8.5 arcsec for the physical pairs. Colour and symbol code is as in Fig. 3. Solid, dashed, and dotted lines indicate the $3 \sigma$ detection limits for primaries in the magnitude ranges $I<10 \mathrm{mag}, 10 \mathrm{mag} \leq I<11 \mathrm{mag}$, and $I \geq 11 \mathrm{mag}$, respectively.

$\Delta I$ using the peak flux value of the star. The maximum magnitude difference in the detection of possible companions at angular separations between 0.2 and 1.0 arcsec varies from 3 to $4 \mathrm{mag}$ and from 5 to $7 \mathrm{mag}$ at separations larger than 2 arcsec, depending on the brightness of the primary star. The limiting magnitude of our survey is about $I \approx 17 \mathrm{mag}$, and we were able to detect all sources brighter than this limit at angular separations greater than 3 arcsec. This implies that at separations larger than 3 arcsec, the detection of companions earlier than M8 dwarfs is complete up to $40 \mathrm{pc}$, which corresponds to the entire sample, and the detection of companions earlier than M9 dwarfs is complete up to $25 \mathrm{pc}$, which is in most of our sample.

\section{Results and discussion}

\subsection{Detected binaries}

Of the 490 observed stars, we confirmed with our data 80 companions in 76 systems, of which 30 are presented here for the first time. In addition, there are also six unconfirmed binaries that need additional epochs to confirm the physical binding. The majority of the optical components of the survey were easily identified using previous available data, and most of the remaining ones were confirmed as physically bound companions using our own measurements at different epochs. Therefore, we considered the six unconfirmed binaries as very probably physically bound rather than unbound pairs. We took into account the six binaries for the determination of the multiplicity fraction.

The 86 pairs are listed in Table A.4. In the last column of the table, we include the multiplicity flag from version 1.2 of the Guide Star Catalog (Morrison et al. 2001), which is "False" for 18 of the 30 new confirmed binaries, "True" for 10 of them and has no entry for the close companions of J08082+211 and J15191-127. For the 30 new binaries, and to our knowledge, there are no other references to binarity.

Of the 80 physical companions, 48 are tabulated by WDS (second column in Table A.4), of which two were previously suggested by Behall \& Harrington (1976; J05333+448) and Bowler et al. (2015; J15496+348) and confirmed here. Another two were recently presented by Ward-Duong et al. (2015; J05034+531) and Ansdell et al. (2015; J06212+442), and one of the new companions resolved here is most likely associated with a spectroscopic binary identified by Bonfils et al. (2013; J15191-127). The remaining 29 are pairs with no previous binarity references to our knowledge.

Some of the measured companions were not detected in all epochs because of the relative motion of the components and the crossing of the companion behind or in front of the primary star (J05078+179 and $\mathrm{J} 05333+448)$, presence of the companion near the diffraction limit $(\mathrm{J} 13317+292$, J15496+348, J16487+106, and $\mathrm{J} 21012+332)$, and a focus problem $(\mathrm{J} 06400+285)$.

\subsection{Multiplicity fraction}

Of the 490 observed M dwarfs, 408 are single and $82(76+6)$ are in binary or multiple systems within the FastCam field of view. This gives a close multiplicity fraction of $16.7 \pm 2.0 \%$, by assuming a Poissonian distribution of the errors. Nevertheless, it must not be taken as a real M-dwarf multiplicity fraction because of the selection bias of the observed sample: we did not include many stars that were previously observed in similar studies or that had known visual companions at less than 5 arcsec.

For statistical purposes, we grouped all our Carmencita (Sect. 1) and FastCam targets in a combined sample. Of the 2176 Carmencita stars, $1141 \mathrm{M}$ dwarfs have been surveyed with FastCam or with high-resolution imagers with similar capabilities (Beuzit et al. 2004; Lafrenière et al. 2007; Law et al. 2008; Bergfors et al. 2010; Janson et al. 2012, 2014a; Jódar et al. 2013; Bowler et al. 2015; Ward-Duong et al. 2015). For completeness, we considered a range of angular separations from 0.2 to 5 arcsec to our targets. The lower limit was given by the FastCam spatial resolution and the upper limit by the maximum separation at which we could detect companions to at least $90 \%$ of the observed stars. Of the 1141 surveyed $\mathrm{M}$ dwarfs, 219 have physical companions in this interval of angular separations (55 from this work and 164 from other publications), which gives a close multiplicity fraction of $19.2 \pm 1.4 \%$.

To avoid any selection bias and give a more reliable multiplicity fraction, we proceeded by building a volume-limited sample with a maximum distance of $14 \mathrm{pc}$ and a completeness of $86 \%$. This completeness was estimated by assuming that all M0-M5 dwarfs are known within $7 \mathrm{pc}$ and that their density in the solar vicinity is constant. This third sample is composed of 425 dwarfs with spectral types between M0.0 V and M5.0 V, of which 83 have companions (either from FastCam and other works) in the range from 0.2 to 5.0 arcsec. This translates into a close multiplicity fraction of $19.5 \pm 2.3 \%$, which is consistent within error bars with the 13.6-27\% fractions obtained for M dwarfs in most surveys (Table 1), although some authors provided higher multiplicity fractions (Fischer \& Marcy 1992; Bergfors et al. 2010). In Sects. 4.8 and 4.9 we estimate the contribution to the multiplicity fraction of pairs separated by less than 0.2 arcsec and more than 5 arcsec.

\subsection{Dependence of multiplicity on spectral type}

To estimate the spectral types of the individual components of the binaries, we used the $I-J$ colours and $M_{I}$ absolute magnitudes as a function of spectral type for M dwarfs from Table 3 in Kirkpatrick et al. (1994), together with the 2MASS photometry and spectral type of the pair.

For pairs resolved by 2MASS, we used these relations and values to obtain the $I$ magnitude of the primary, and obtained the $I$ magnitude of the secondary from our measured $\Delta I$. We 


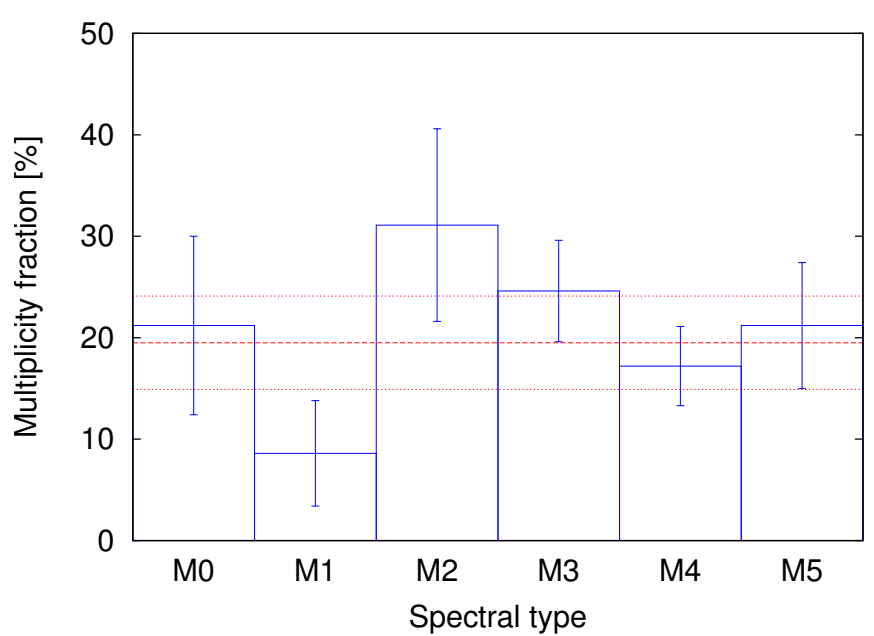

Fig. 5. Multiplicity fraction as a function of spectral type from M0.0 $\mathrm{V}$ to $\mathrm{M} 5.0 \mathrm{~V}$ in the volume-limited sample with separations from 0.2 to 5.0 arcsec. Error bars are Poissonian. Horizontal dashed and dotted lines are the global multiplicity fraction and the $\pm 2 \sigma$ values.

derived the absolute $M_{I}$ magnitudes through the distance modulus and inferred the spectral types of the secondaries with the $M_{I}$-spectral type relation of Kirkpatrick et al. (1994).

For pairs not resolved by 2 MASS, the $J$-band magnitude involves the contribution of all the components in the system. In these cases, we obtained the $I$ magnitude of the system from the $I-J$ colours and the global spectral types of the pairs from the literature. Using the $I$ magnitude and our measured $\Delta I$, we computed the individual $I$ magnitudes. We calculated the individual $M_{I}$ absolute magnitudes by applying the distance modulus, and estimated individual spectral types from the $M_{I}$-spectral type relation.

The distances in our sample come mostly from literature parallax determinations (see references in Table A.1). For stars without parallactic distance, we calculated spectro-photometric distances from our own $M_{J}$-spectral type relation. This relation was obtained from a polynomial fit using single stars with welldetermined spectral types between M0 V and M6 V, parallactic distances, and 2MASS $J$-band photometry from the Carmencita sample, and has the form:

$M_{J}=a \mathrm{SpT}^{2}+\mathrm{bSpT}+\mathrm{c}$,

where $a=0.078 \pm 0.007 \mathrm{mag}, \quad b=0.265 \pm 0.038 \mathrm{mag}$ and $c=5.895 \pm 0.044 \mathrm{mag}$, and SpT indicates the numerical spectral subtype within the $M$ range.

For very close binaries, spectro-photometric distances are not reliable since the 2MASS photometry and the spectral type determination do not provide the contribution of the two components separately. In these cases, in an iterative way, we estimated new individual spectro-photometric distances for the two components in the system from spectral type estimations based on the global spectral type, the $M_{I}$-spectral type relation, the individual $I$ magnitudes, and the distance modulus. These updated distances are given in Table A.1. Given the low number of close binaries not resolved in our survey $(\sim 10 \%$, see Sect. 4.8$)$, we do not expect many additional unresolved components.

The individual spectral types are listed in Table A.5. SpT column indicates the combined spectral type of the system from which individual spectral types were derived. In the $\mathrm{SpT}_{1}$ and $\mathrm{SpT}_{2}$ columns, the spectral types indicated with capital " $\mathrm{M}$ " come from the literature, and with lower case " $m$ " refer to our estimated spectral types.

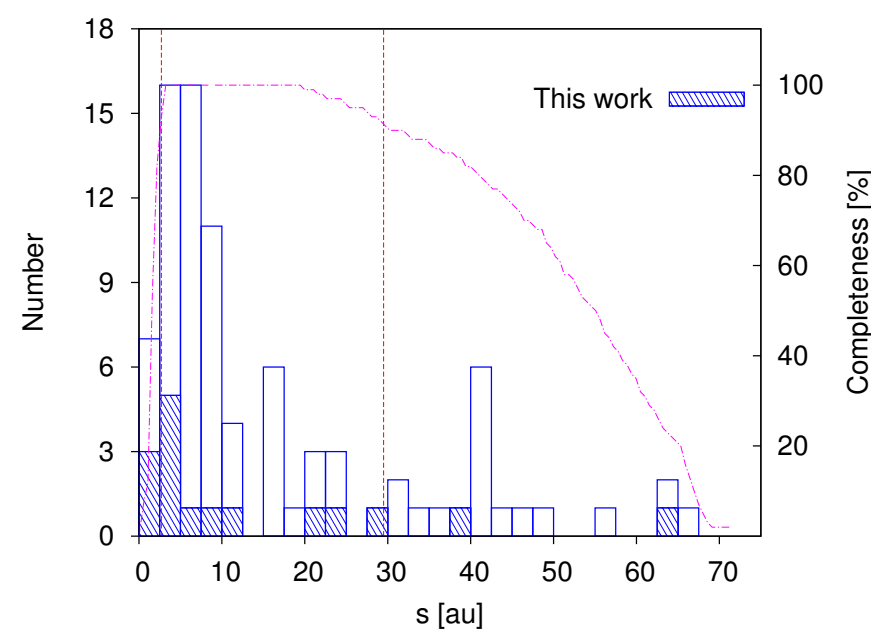

Fig. 6. Projected physical separation distribution of the binaries in the volume-limited sample. Dashed bars represent our binaries. Vertical dashed lines mark the $90 \%$ completeness limits, and the dash-dotted curve represents the completeness as a function of the projected physical separation.

In Fig. 5 we show the dependence of the multiplicity fraction of $\mathrm{M}$ dwarfs on the spectral type in our volume-limited sample. The multiplicity fractions for different spectral subtypes are consistent within the error bars among them, except for M1 stars, for which it is lower. We compared this distribution with the global multiplicity fraction obtained in the previous section and performed a $\chi^{2}$ test. Without the M1 contribution, the distribution is consistent with a flat distribution with a confident level of $96 \%$.

In addition, our determined multiplicity fraction has intermediate values between Sun-like (44-65\%) and very low mass stars and brown dwarfs $(9-15 \%)$. This agrees with the generally accepted decreasing trend of the multiplicity fraction with decreasing mass of the primaries (Table 1).

\subsection{Projected physical separation distribution}

To study the distribution of the binaries in the volume-limited sample, we converted angular separations $(\rho)$ into projected physical separations $(s)$ by using the small-angle approximation $\tan \rho \approx \rho$. Hence, $s \approx \rho d$. The distances $d$ come from parallax or photometry as in Sect. 4.3.

In Fig. 6 we show the projected physical separation distribution of the binaries in the volume-limited sample. We also represent the completeness of the volume-limited sample as a function of projected physical separation, and draw the completeness limits with a confidence level of $90 \%$, which correspond to the $s$ interval between 2.6 and $29.5 \mathrm{au}$. We estimated these values as those separations at which we are able to detect companions in $90 \%$ of the sample.

The projected physical separations of the observed pairs in the volume-limited sample range from 1.4 to $65.6 \mathrm{au}$ and their distribution peaks at $2.5-7.5 \mathrm{au}$. This is consistent with the values of 5-10 au found by Jódar et al. (2013) for M0-M4 dwarfs, and of $\sim 6$ au found by Janson et al. (2014) for M3-M8 dwarfs and Ward-Duong et al. (2015) for M0-M6 dwarfs. However, these values are lower than those found for Sun-like stars (Duquennoy \& Mayor 1991; Raghavan et al. 2010) and more similar to those found for ultracool dwarfs (4-6 au for M8.0-L0.5, Close et al. 2003; 2-4 au for M7.0-L8.0, Bouy et al. 2003; <3 au for L dwarfs, Reid et al. 2008). 
Table 3. Target members of stellar kinematic groups.

\begin{tabular}{llllll}
\hline \hline Karmn & Name & $\begin{array}{l}\text { Moving } \\
\text { group }\end{array}$ & Ref. $^{a}$ & $\begin{array}{l}\text { Assumed } \\
\text { age [Ma }]\end{array}$ & Ref. $^{b}$ \\
\hline J01221+221 & G 034-023 & Young disc & Abe14 & $\geq 300$ & This work \\
J04153-076 & $o^{02}$ Eri C & $\beta$ Pic & AF15 & $\sim 20$ & Bell15 \\
J05019+099 & LP 476-207 & $\beta$ Pic & AF15 & $\sim 20$ & Bell15 \\
J05068-215E & BD-21 1074 A & $\beta$ Pic & AF15 & $\sim 20$ & Bell15 \\
J05068-215W & BD-21 1074 BC & $\beta$ Pic & AF15 & $\sim 20$ & Bell15 \\
J05103+488 & G 096-021 AB & IC 2391? & This work & $\sim 50$ & Barr04 \\
J10028+484 & G 195-055 & Local Association? & This work & $\sim 100$ & Bas96 \\
J10196+198 & BD+20 2465 & Castor & Cab10 & $\geq 300$ & Barr98, Mam13 \\
J12123+544S & BD+55 1519 A & UMa & Mon01 & $\geq 300$ & Gia79, SM93 \\
J12123+544N & BD+55 1519 B & UMa & Mon01 & $\geq 300$ & Gia79, SM93 \\
J13317+292 & DG CVn AB & Columba/Carina & Ried14 & $\sim 40$ & Bell15 \\
J18548+109 & V 1436 Aql B & Castor & Cab10 & $\geq 300$ & Barr98, Mam13 \\
J23293+414S & G 190-027 & Local Association & Klu14 & $\sim 100$ & Bas96 \\
J23293+414N & G 190-028 & Local Association & Klu14 & $\sim 100$ & Bas96 \\
J23318+199 E & EQ Peg Aab & Castor & Cab10 & $\geq 300$ & Barr98, Mam13 \\
J23318+199 W & EQ Peg Bab & Castor & Cab10 & $\geq 300$ & Barr98, Mam13 \\
\hline
\end{tabular}

Notes. ${ }^{(a)}$ Abe14: Aberasturi et al. (2014); AF15: Alonso-Floriano et al. (2015); Cab10: Caballero (2010); Klu14: Klutsch et al. (2014); Mon01: Montes et al. (2001); Ried14: Riedel et al. (2014). ${ }^{(b)}$ Barr04: Barrado y Navascués et al. (2004); Barr98: Barrado y Navascués (1998); Bas96: Basri et al. (1996); Bell15: Bell et al. (2015); Gia79: Giannuzzi (1979); Mam13: Mamajek et al. (2013); SM93: Soderblom \& Mayor (1993).

Within the physical separation completeness range from 2.6 to $29.5 \mathrm{au}$, there are $61 \mathrm{M}$ dwarfs with low-mass companions in our volume-limited sample. This translates into a multiplicity fraction of $14.4 \pm 2.0 \%$, which is lower than the fraction derived in Sect. 4.2 as a result of the missing systems at larger separations (see Fig. 6).

\subsection{Masses}

We derived masses from our own mass-luminosity relation in the Johnson-Cousins $I$ band. To our knowledge, there is no published mass-luminosity relation employing this band. We collected dynamical masses and I-band magnitudes of eleven low-mass stars from different works (Delfosse et al. 2000; Henry 2004; Reid et al. 2004; Tokovinin 2008) and obtained an $M_{I}-\mathcal{M}$ relation using a parabolic fit of the form:

$\log \mathcal{M}=a M_{I}^{2}+b M_{I}+c$,

where $\mathcal{M}$ is the mass, $M_{I}$ is the absolute $I$-band magnitude, $a=0.005 \pm 0.002 \mathrm{mag}^{-2}, b=-0.222 \pm 0.037 \mathrm{mag}^{-1}$, and $c=1.035 \pm 0.180$. This relation is valid for main-sequence stars in the $M_{I}$ interval between 6 and $14 \mathrm{mag}$, which corresponds to $\sim \mathrm{M} 0-\mathrm{M} 8$ spectral types. Figure 7 shows the data taken from the literature, the corresponding best fit, and the comparison with BT-Settl evolutionary models from the Lyon group (Baraffe et al. 2015).

In some of our detected pairs, one or both components are also spectroscopic binaries (see Table 6). For these we estimated individual masses assuming equally bright components.

For main-sequence stars, the luminosity and effective temperatures are unambiguosly related to the mass, and thus, the relation in Eq. (3) is only valid for stars older than $\sim 300 \mathrm{Ma}$, as

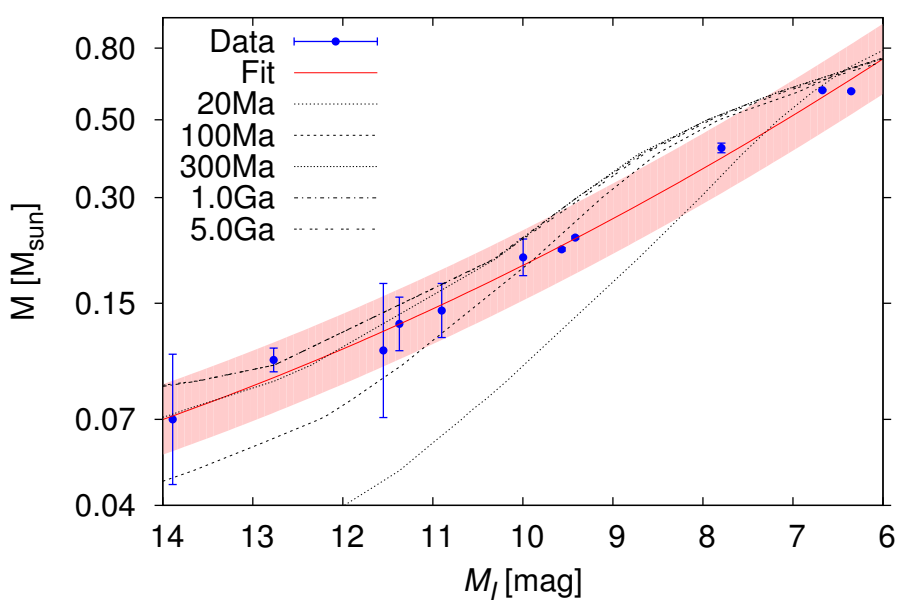

Fig. 7. Mass $\mathcal{M}$ vs. Absolute magnitude $M_{I}$. Blue points represent the dynamical masses and absolute magnitudes taken from the literature. The red solid line and shadowed area represent the best-fit $\pm 3 \sigma$. Different dashed lines display the BT-Settl evolutionary models at $20 \mathrm{Ma}$, $100 \mathrm{Ma}, 300 \mathrm{Ma}, 1 \mathrm{Ga}$, and $5 \mathrm{Ga}$.

inferred from Fig. 7. For stars younger than $300 \mathrm{Ma}$, the massluminosity relation strongly depends on the age. We searched for young stars in our sample by collecting radial velocities from the literature (Caballero et al., in prep.) and computing UVW Galactocentric space velocities as in Montes et al. (2001) for 452 of the 490 observed stars (there are 38 stars without radial velocities). Of these, 155 have $U$ and $V$ velocity components inside or near the boundaries that delineate the young-disc population (Montes et al. 2016). In total, 42 stars of our 82 multiple systems are candidate members in young stellar kinematic groups. We checked 
the literature and found that 26 of the 42 are relatively old interloper stars that do not show any youth feature or have been poorly investigated. The remaining 16 stars are confirmed members of stellar kinematic groups or the young-disc population. Their associations and ages are listed in Table 3.

Since $I-J$ colours of young stars and field stars do not show significant differences (see Bihain et al. 2010; Peña-Ramírez et al. 2016), we applied the colour-spectral-type relation from Kirkpatrick et al. (1994) to derive the individual I magnitudes of these stars as explained in Sect. 4.3. We considered Castor, Ursa Majoris, and young-disc members old enough to be mainsequence stars, and thus, to apply our mass- $M_{I}$ relation with confidence. For these calculations, we assumed the ages given in Table 3. These stars appear in italics in Table A.5. The candidate pair to IC 2391 does not have a parallactic distance. Hence, we estimated its mass from the $I-J$ colours and the BTSettl evolutionary models from the Lyon group (Baraffe et al. 2015). We also applied these models to derive masses from the individual $I$ magnitudes and parallactic distances for $\beta$ Pic, Columba/Carina and Local Association members.

Table A.5 lists the inferred I magnitudes (Sect. 4.3) and mass values of the components of 76 of our systems. All of the detected companions have absolute magnitudes brighter than 14 mag, the lowest limit of our empirical mass-magnitude relation, which corresponds to masses close to the hydrogen-burning limit $\left(\sim 0.07 M_{\odot}\right)$. The only exception is the unconfirmed companion of J04352-161, which has an absolute magnitude fainter than $14 \mathrm{mag}$, and we were unable to determine its mass with the method explained before.

\subsection{Mass ratios}

The upper panel in Fig. 8 shows the mass ratio $\left(\mathcal{M}_{2} / \mathcal{M}_{1}\right)$ histogram of our binaries in Table A.5. This global distribution slightly increases towards higher mass ratios and has its maximum above 0.8 . The slightly lower number of equal-mass pairs with mass ratios near unity is not significant and could be related to the effect of the reduction process using the brightest pixel, which artificially sharpens the PSF of the primary with respect to the PSF of the secondary, and may produce a lower flux ratio than expected. This distribution is also affected by our sensitivity limit. While in spectral types earlier than M3.5 (i.e. more massive stars) our search of companions is complete for mass ratios greater than 0.3 , in later spectral types (i.e. less massive stars) the search is complete for mass ratios greater than $0.35-0.60$.

Empty and dashed bars represent the mass ratio distributions of M0.0-M3.5 and M4.0-M5.5 primaries, respectively. The distribution of the former shows the same trend as the global distribution, with a peak around 0.8-0.9. For the latter, the distribution increases towards higher ratios. As explained before, this might be due to our observational bias.

The high occurrence of binaries with mass ratios above 0.8 can also be seen in the lower panel in Fig. 8, which represents the spectral type of the primary versus the mass ratio. For later spectral types, our detected binaries also tend to have similar masses. This may be due to the lack of sensitivity to lower mass ratios at later spectral types. The distribution differs with the more homogeneous mass ratio distributions observed by Janson et al. (2012, 2014). The number of binaries with mass ratios closer to unity (i.e. similar masses) for M0.0-M3.5 contrasts with the relatively low numbers presented in Bergfors et al. (2010) in this range, but is more similar to their distribution for later M4.0-M5.5 spectral types.
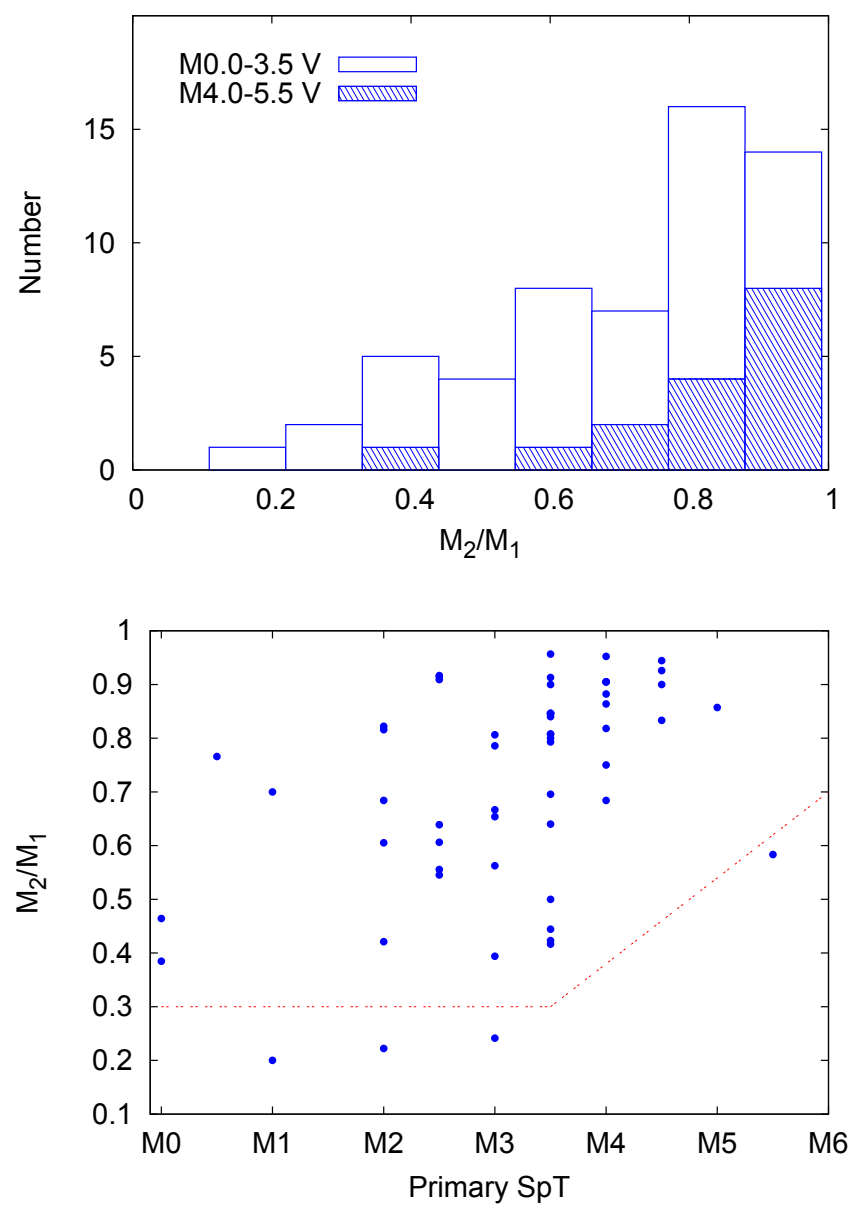

Fig. 8. Top panel: mass ratio distribution of our binaries. Empty and dashed bars separate the mass ratio distribution of M0.0-M3.5 and M4.0-M5.5 dwarfs. Bottom panel: mass ratio of the pairs vs. spectral type of the primary. The red dashed line represents the mass ratio completeness limits. The standard error of the mean mass ratio is 0.03 and the error bar is \pm 0.5 in spectral type.

Figure 9 displays the occurrence of mass ratios with physical separations. Pairs with separations shorter than 50 au tend to have mass ratios over 0.8 , while pairs at larger separations present a more homogeneous distribution.

Similar studies also show this observed trend in the relation between separation of the components and mass ratio: near equal-mass pairs (mass ratios $\geq 0.8$ ) are found at smaller separations. Moverover, the lower the mass of the primary, the higher the mass ratio and the closer the semi-major axis at which companions are found (Jódar et al. 2013: Janson et al. 2012 , 2014). The closer distance to the Sun of our sample compared to the samples of Bergfors et al. (2010) and Janson et al. (2014), who investigated the mass ratio at larger separations, may explain the difference with our results in the mass ratio distribution. However, Monte Carlo simulations of Sun-like stars and M-dwarf surveys from Duquennoy \& Mayor (1991) and Raghavan et al. (2010), and Fischer \& Marcy (1992) and Janson et al. (2012), respectively, suggest that the mass ratio distributions could be independent of the separation and dynamical evolution (Reggiani \& Meyer 2011, 2013).

\subsection{Periods and orbital motion}

We derived periods for 70 systems with Kepler's third law, the masses of the components, and the maximum projected physical 


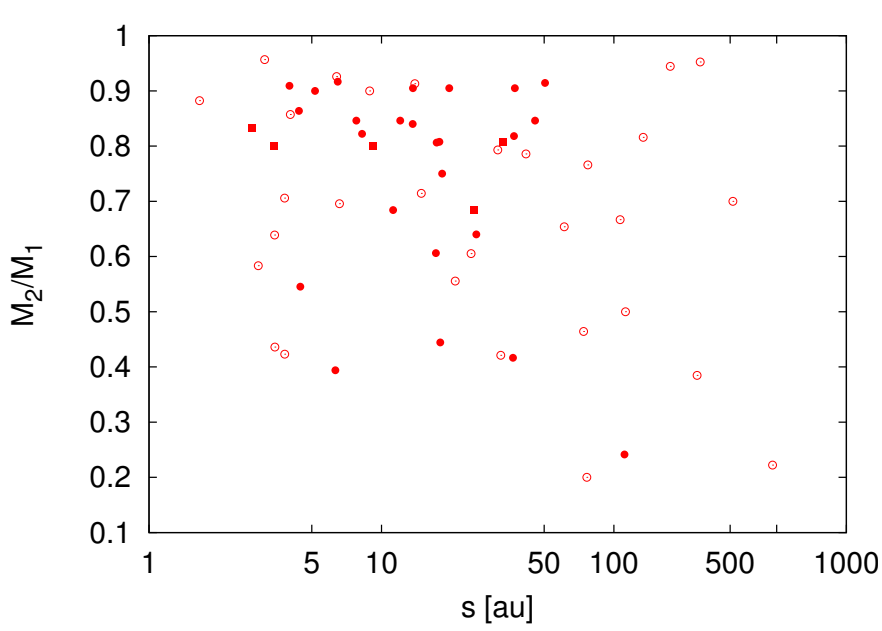

Fig. 9. Mass ratio vs. projected physical separation. Colour and symbol code is as in Fig. 3.

separations (Sect. 4.4). Since these measures are a lower limit estimate to the semi-major axis, the periods given in Table A.5 should be also considered as a lower limit.

In total, 26 systems have periods shorter than $50 \mathrm{a}$, of which 13 are known bound systems, 10 are newly discovered binaries, and three are the unconfirmed pairs J01221+221, J07349+147, and $\mathrm{J} 10028+484$.

Of the 26 systems, we consider four triple systems here: $\mathrm{J} 05078+179$, J08082+211, and J16554-083S, which are formed by a spectroscopic binary plus a third resolved component, and $\mathrm{J} 23293+414 \mathrm{~S}$, for which we resolved the three components of the system. In addition, the "triples" J08082+211 and J16654-083S belong to a hierarchical quadruple and quintuple system, respectively, with the fourth and fifth components outside the field of view of FastCam (Sect. 4.9).

Several systems were observed repeatedly during the programme, which allowed us to perform a multi-epoch analysis. Some of them showed appreciable variation of angular separation and position angle in different epochs of our data. When these variations were larger than $3 \sigma$ with respect to constant values of $\rho$ and $\theta$ and were consistent with an orbital trajectory, we considered that the orbital motion of the pair was detected. Because of the large uncertainties, the variations of $\rho$ and $\theta$ of the pairs J05333+448, J08066+558, and J20407+199 lie within $3 \sigma$ and therefore they do not fulfil our criterion, but they show appreciable variations that are probably related to the orbital motion. However, the time baseline is not long enough to provide a precise estimate of the orbital parameters of the systems.

Table 4 lists these 16 systems, of which 13 are new. We tabulate the WDS discoverer code of the previously known pairs, the number of used epochs, the time interval between the first and last measured epoch, and the estimated periods. We show an example of one of these binaries (J12332+090) in Fig. 10.

\subsection{Known close and spectroscopic binaries (not detected in our search)}

In the observed sample there were also previously known pairs that we were unable to resolve because of the small separation of the components ( $\rho \lesssim 0.2 \operatorname{arcsec}$ ) and/or the faintness of the companion. These pairs are listed in Table 5. In addition, there were also previously known spectroscopic binaries, taken into account for the period estimation of our detected binaries in Table A.5. They are listed in Table 6.

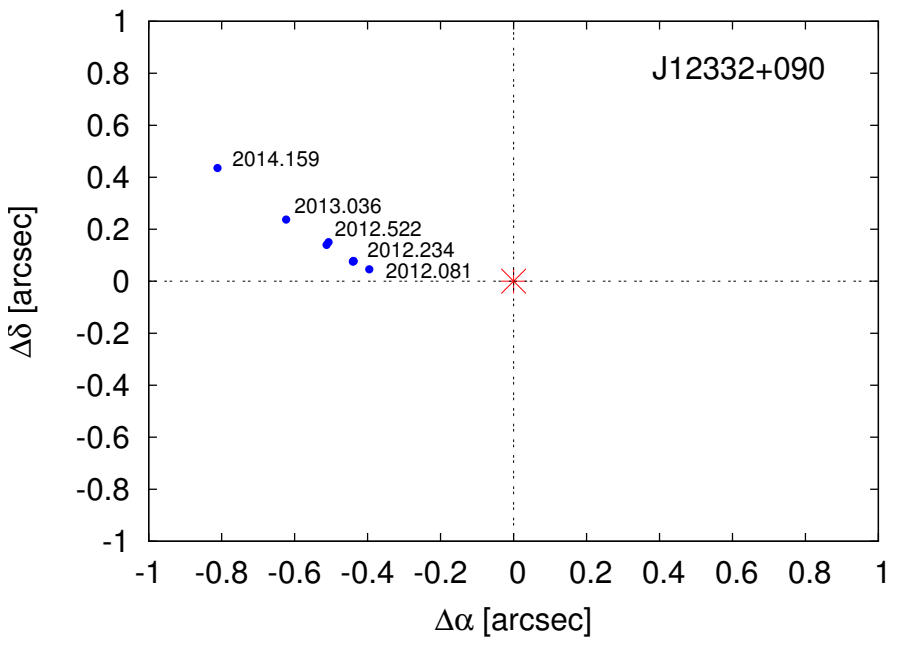

Fig. 10. Orbital variation of the pair $\mathrm{J} 12332+090$ from our FastCam data. The asterisk marks the position of the primary. Five of the eight epochs are labelled.

Table 4. Systems with measurable orbital motion.

\begin{tabular}{lcccc}
\hline \hline Karmn & WDS & Epochs & $\begin{array}{c}\Delta t \\
{[\mathrm{a}]}\end{array}$ & $\begin{array}{c}P \\
{[\mathrm{a}]}\end{array}$ \\
\hline J02518+294 & $\ldots$ & 3 & 4.2 & 130 \\
J05068-215W & DON 93 & 3 & 1.2 & 62 \\
J05078+179 & $\ldots$ & 2 & 1.1 & 50 \\
J05333+448 & BH 76 & 6 & 2.3 & 8.3 \\
J06400+285 & $\ldots$ & 3 & 1.0 & 20 \\
J08066+558 & $\ldots$ & 4 & 3.2 & 26 \\
J08082+211 & $\ldots$ & 2 & 3.8 & 33 \\
J08595+537 & $\ldots$ & 2 & 1.1 & 19 \\
J11355+389 & $\ldots$ & 5 & 3.0 & 31 \\
J11521+039 & $\ldots$ & 2 & 1.1 & 15 \\
J12332+090 & REU 1 & 8 & 2.1 & 16 \\
J13180+022 & $\ldots$ & 4 & 3.1 & 73 \\
J14210+275 & $\ldots$ & 2 & 3.0 & 97 \\
J16487+106 & $\ldots$ & 3 & 1.1 & 10 \\
J17530+169 & $\ldots$ & 5 & 3.0 & 110 \\
J20407+199 & RAO 23 & 2 & 2.8 & 8.4 \\
J21518+136 & $\ldots$ & 3 & 3.0 & 66 \\
\hline
\end{tabular}

The close multiplicity fraction of $19.5 \pm 2.3 \%$ given in Sect. 4.2 is a lower limit of the total multiplicity fraction of M dwarfs, since it only includes physical companions in the interval of angular separations between 0.2 and 5.0 arcsec. Although studies of spectroscopic binaries and very close binaries ( $\rho<0.2$ arcsec) are not complete, we know from the literature that we are missing 47 very close additional binaries in this range in our volume-limited sample (e.g. Delfosse et al. 2013; Schöfer et al. 2015; Tokovinin et al. 2015). This number is consistent with the fractional incidence of eclipsing binaries obtained from surveys like Kepler (Shan et al. 2015), and increases the given binary fraction by $11 \%$. Hence the multiplicity fraction at separations smaller than 5 arcsec would be at least $\sim 30 \%$.

\subsection{Known companions at separations larger than 5 arcsec}

Many of our FastCam stars have stellar or substellar companions outside the field of view of the instrument or at angular separations larger than the 5.0 arcsec cut-off defined for statistical 
M. Cortés-Contreras et al.: High-resolution imaging of CARMENES M dwarfs with FastCam

Table 5. Astrometric properties of previously known imaging companions at $\rho<5 \operatorname{arcsec}$ not resolved or detected in our data.

\begin{tabular}{lccccccc}
\hline \hline Karmn & WDS & $\begin{array}{c}\text { Discoverer } \\
\text { code }\end{array}$ & $\begin{array}{c}\rho \\
{[\text { arcsec }]}\end{array}$ & $\begin{array}{c}\theta \\
{[\mathrm{deg}]}\end{array}$ & $\begin{array}{c}\text { Epoch } \\
{[\mathrm{a}]}\end{array}$ & $\begin{array}{c}\text { Ref. }^{a} \\
\Delta \text { mag }^{(\mathrm{band})} \\
{[\mathrm{mag}]}\end{array}$ \\
\hline J00088+208 & $00089+2050$ & BEU 1 & 0.133 & 271.9 & 2012.02 & Jan14a & $1.59\left(i^{\prime}\right)$ \\
J05085-181 & $05086-1810$ & WSI 72 & 0.07 & 44.4 & 2011.04 & WD15 & $0.1\left(K_{\mathrm{s}}\right)$ \\
J04311+589 & $\ldots$ & $\ldots$ & 0.07 & & 1965.702 & Str77 & $0.5(V)$ \\
J06523-051 & $06523-0510$ & WSI 125 & 0.18 & 149.6 & 2010.068 & Mas01 & $0.5(\mathrm{o})$ \\
J07307+481 & $\ldots$ & $\ldots$ & 0.054 & $\ldots$ & 1960.60 & Harr81 & $\ldots$ \\
J09177+462 & $09177+4612$ & JNN 68 & 0.204 & 37.5 & 2011.073 & Bow15 & $0.102\left(K_{\mathrm{s}}\right)$ \\
J10513+361 & $10513+3607$ & BWL 26 & 0.206 & 119.60 & 2012.357 & Bow15 & $3.3(H)$ \\
J12290+417 & $12290+4144$ & BWL 31 & 0.0503 & 255.5 & 2011.469 & Bow15 & $0.647(H)$ \\
J16241+483 & $16240+4822$ & HEN 1 & 0.1387 & 295.4 & 2006.62 & Mar07 & $2.781\left(H_{\text {cont }}\right)$ \\
J16354+350 & $16355+3501$ & BWL 44 & 0.092 & 25.62 & 2011.469 & Bow15 & $0.406(H)$ \\
J17177+116 & $\ldots$ & $\ldots$ & $\ldots$ & $\ldots$ & 1977 & Chr78 & $\ldots$ \\
J18387-144 & $18387-1429$ & HDS 2641 & 0.107 & 358 & 1991 & DN00 & $0.04(H p)$ \\
J19122+028 & $19121+0254$ & AST 1 & 0.16 & 319.7 & 2007.36 & WD15 & $0.80(H)$ \\
J20298+096 & $20298+0941$ & AST 2 & 0.160 & 89.1 & 2012.66 & Jan14a & $2.72\left(z^{\prime}\right)$ \\
J20433+553 & $20433+5521$ & LLO 1 & 0.854 & 20.2 & 2007.66 & Ire08 & $5.06(H)$ \\
J21013+332 & $21013+3314$ & JNN 288 & 0.142 & 34.0 & 2012.01 & Jan14a & $1.07\left(i^{\prime}\right)$ \\
J21160+298E & $21161+2951$ & BWL 56 & 0.0543 & 354.6 & 2011.47 & Bow15 & $0.37(H)$ \\
J21313-097 & $21313-0947$ & BLA 9 & 0.16 & 128.2 & 2005.33 & WD15 & $1.12(H)$ \\
J23174+196 & $23175+1937$ & BEU 23 & 0.145 & 220.2 & 2012.65 & Jan14b & $1.17(J)$ \\
\hline
\end{tabular}

Notes. ${ }^{(a)}$ Bow15: Bowler et al. (2015); Chr78: Christy (1978); DN00: Dommanget \& Nys (2000); Harr81: Harrington et al. (1981); Ire08: Ireland et al. (2008); Jan14a: Janson et al. (2014a); Jan14b: Janson et al. (2014b); Mar07: Martinache et al. (2007); Mas01: Mason et al. (2001); Str77: Strand (1977); Tok15: Tokovinin et al. (2015); WD15: Ward-Duong et al. (2015). ${ }^{(b)}$ The BWL 44 companion at 2.2 arcsec is optical. ${ }^{(c)}$ Astrometric perturbation with a 10 a period estimation in Chr78. ${ }^{(d)}$ Spectroscopic binary identified by Benedict et al. (2000) and resolved by Janson et al. (2014a) for the first time.

purposes. We compiled the multiplicity information of all of them using our observations and the WDS catalogue. Of the wide binaries present in the WDS catalogue, nearly $60 \%$ come from the Luyten Double Star Catalogue (Luyten 1997) and the Lowell Proper Motion Survey (Giclas et al. 1971). In Table A.6, we list for each wide system the WDS discoverer code, names, spectral types, and angular separation.

As a summary, of the 490 observed stars, 50 are M-dwarf primaries with M-type wide companions, four with white dwarf companions, one with an L-dwarf, and three with a T-dwarf secondary. In addition, $11 \mathrm{M}$ secondaries have F(2), G (3), K (4) or white dwarf (3) primaries. Five tertiary $M$ dwarfs are in triple systems involving $\mathrm{K}+\mathrm{M}(1), \mathrm{G}+\mathrm{K}(1), \mathrm{K}+\mathrm{DA}(1)$ or $\mathrm{K}+\mathrm{K}(2)$ primaries.

In our volume-limited sample are $25 \mathrm{M}$ dwarf primaries with wide $\mathrm{M}, \mathrm{L}$, or $\mathrm{T}$ dwarf secondaries at separations larger than 5 arcsec. Although our search at wide separations is not complete, since we carried out a compilation from different studies in the literature, we estimated an increment in the multiplicity fraction of $6 \%$ (25 systems out of $425 \mathrm{M}$ dwarfs in our volumelimited sample), which added to the percentage estimated for pairs at separations closer than 0.2 arcsec, and spectroscopic binaries would translate into a minimum multiplicity fraction at all separations of $\sim 36 \%$.

\section{Summary}

We obtained high-resolution images in the $I$ band of $490 \mathrm{M}$ dwarfs of the CARMENES input catalogue (Carmencita) with the lucky imaging instrument FastCam at the $1.5 \mathrm{~m}$ Telescopio Carlos Sánchez.
Among the 490 observed M dwarfs, we identified 80 physically bound companions in 76 systems, of which 30 are presented here for the first time, plus six unconfirmed companions. For all of them, we measured angular separations, position angles, and $I$-band magnitude differences. From the $\Delta I$ differences, together with 2MASS photometry, spectral type, and colour-magnitude relations for field $\mathrm{M}$ dwarfs, we estimated individual $I$-band magnitudes and spectral types of each component. We also derived individual masses $\mathcal{M}$ and estimated orbital periods for these pairs from our own $\mathcal{M}-M_{I}$ relation. For these calculations, we used parallactic distances. When not available, we derived spectro-photometric distances from our determined $M_{J}$-spectral type relation.

For our observed sample, we determined a multiplicity fraction of $16.7 \pm 2.0 \%$. However, our sample has a strong selection bias because we discarded M stars with previously known companions at separations smaller than 5 arcsec. To obtain an unbiased multiplicity fraction, we built a volume-limited sample of Carmencita stars observed with FastCam and similar highresolution imagers. It contains $425 \mathrm{M} 0-5$ dwarfs and is complete up to $86 \%$ within $14 \mathrm{pc}$. For this sample, we derived a multiplicity fraction of $19.5 \pm 2.3 \%$ in the completeness range of angular separations between 0.2 and 5.0 arcsec, which agrees with previously reported values (Leinert et al. 1997; Janson et al. 2012, 2014; Jódar et al. 2013; Ward-Duong et al. 2015). The multiplicity fraction is consistent with a flat distribution from M0 V to M5 V within Poissonian error bars, and has intermediate values between solar-type stars and very low mass stars and brown dwarfs in accordance with the decreasing tendency observed towards lower masses.

The distribution of the number of pairs as a function of projected physical separation has a maximum between 2.5 and 
Table 6. Known spectroscopic binaries in the observed sample.

\begin{tabular}{lcc}
\hline \hline Karmn & $\begin{array}{c}\text { Spectroscopic } \\
\text { binarity }\end{array}$ & Ref $^{a}$ \\
\hline J03346-048 & SB3 & Llam14 \\
J03526+170 & SB2 & Bon13 \\
J04252+080S & SB2 & Llam14 \\
J04352-161 & SB2 & RB09 \\
J04488+100 & SB2 & Jeff16 \\
J05019+099 & SB2 & De199 \\
J05032+213 & SB2 & Jeff16 \\
J05078+179 & SB1 & Jeff16 \\
J05342+103S $b$ & SB & Rein12 \\
J05466+441 & SB2 & Jeff16 \\
J07418+050 & SB2 & Llam14 \\
J08082+211 & SB2 & Shk10 \\
J09011+019 & SB2 & Jeff16 \\
J09120+279 & SB2 & Jeff16 \\
J09143+526 & SB1 & Jeff16 \\
J11036+136 & SB1 & Jeff16 \\
J12142+006 & SB2 & Bon13 \\
J12191+318 & SB2 & Jeff16 \\
J12290+417 & SB2 & Jeff16 \\
J14171+088 & SB2 & Jeff16 \\
J14368+583 & SB2 & Jeff16 \\
J15191-127 & SB & Bon13 \\
J16255+260 & SB2 & Jeff16 \\
J16487+106 & SB2 & Jeff16 \\
J16554-083S & SB & Pett84 \\
J18411+247S & SB2 & GR96 \\
J19354+377 & SB1 & Jeff16 \\
J20433+553 & SB2 & Ire08 \\
J20445+089N & SB1 & Jeff16 \\
J23096-019 & SB2 & Jeff16 \\
J23174+382 & SB2 & Jeff16 \\
J23318+199E & SB1 & De199 \\
J23318+199W & SB1 & Del99 \\
J23573-129W & SB2 & Jeff16 \\
\hline & & \\
\hline
\end{tabular}

Notes. ${ }^{(a)}$ Bon13: Bonfils et al. (2013); Del99: Delfosse et al. (1999); GR96: Gizis \& Reid (1996); Ire08: Ireland et al. (2008); Jeff16: Jeffers et al. (in prep.); Llam14: Llamas 2014; Pett84: Pettersen et al. (1984); RB09: Reiners \& Basri (2009); Rein12: Reiners et al. (2012); Shk10: Shkolnik et al. $\left(2010{ }^{(b)}\right.$ From the spectral types and magnitude differences of the components, we infer that the spectroscopic binary is the B companion. ${ }^{(c)}$ Equal-brightness close binary previously suggested by Cortés-Contreras et al. (2014).

$7.5 \mathrm{au}$ and decreases at wider separations. The pairs with projected physical separations smaller than 50 au tend to have mass ratios higher than 0.8 , while for larger separations this distribution is more uniform.

We estimated that 26 of our systems have orbital periods shorter than 50 a, of which 10 are newly discovered systems. In 17 of them, we were able to detect orbital variations within our own multi-epoch measurements. These systems are especially interesting for future astrometric follow-up for determining their orbital solutions and measuring dynamical masses.

For our volume-limited sample, we also collected from the literature the physically bound companions at separations closer than 0.2 arcsec and larger than 5 arcsec, and unresolved spectroscopic binaries. The addition of these systems may increase the multiplicity fraction derived in this work to at least $36 \%$, a value consistent with the $42 \pm 9 \%$ obtained by Fischer \& Marcy (1992). Nevertheless, the sample is not complete at separations beyond the completeness limit of our survey $(0.2-5.0$ arcsec) and, hence, this value must only be considered as a rough estimation.

Finally, we provided a complete sample of multiple M dwarfs useful for studying the effect of low-mass stellar multiplicity on planet formation with the help of CARMENES and other near-infrared high-resolution spectrographs.

Acknowledgements. We thank A. Pérez-Garrido for the provision and support of the FastCam reduction software and X. Bonfils for the supply of radial velocity measurements from the ESO HARPS GTO Program ID 072.C-0488. M.C.C. thanks L. Peralta de Arriba, V. Pereira and H. M. Tabernero for their assistance and valuable conversations. This article is based on observations made with the Telescopio Carlos Sánchez operated on the island of Tenerife jointly by the Instituto de Astrofísica de Canarias and the Universidad de La Laguna in the Spanish Observatorio del Teide. This research made use of SIMBAD, operated at Centre de Données astronomiques de Strasbourg (France), the NASA's Astrophysics Data System, the Washington Double Star catalogue (WDS) maintained at the US Naval Observatory, and the Image Reduction and Analysis Facility (IRAF), distributed by the National Optical Astronomy Observatory and operated by the Association of Universities for Research in Astronomy (AURA) under a cooperative agreement with the National Science Foundation. CARMENES is funded by the German Max-Planck-Gesellshaft (MPG), the Spanish Consejo Superior de Investigaciones Científicas (CSIC), the European Union through FEDER/ERF funds, and the members of the CARMENES Consortium (Max-Planck Institut für Astronomie, Instituto de Astrofísica de Andalucía, Landessternwarte Königstuhl, Institut de Ciènces de l'Espai, Institut für Astrophysik Göttingen, Universidad Complutense de Madrid, Thüringer Landessternwarte Tautenburg, Instituto de Astrofísica de Canarias, Hamburger Sternwarte, Centro de Astrobiología, and the Centro Astronómico Hispano-Alemán), with additional contributions by the Spanish Ministry of Economy, the state of Niedersachsen, the German Science Foundation (DFG), and by the Junta de Andalucía. Financial support was also provided by the Junta de Andalucía, and the Spanish Ministries of Science and Innovation and of Economy and Competitiveness, under grants 2011-FQM-7363, AP2009-0187, AYA2014-54348-C3-01/02/03-R, AYA201569350-C3-2-P, ESP2013-48391-C4-1-R, and ESP2014-57495-C2-2-R.

\section{References}

Aberasturi, M., Caballero J. A., Montesinos B., et al. 2014, AJ, 148, 36

Aitken, R. G., \& Doolittle, E. 1932, New general catalogue of double stars within $120^{\circ}$ of the north pole, Carnegie Institution of Washington, USA

Alonso-Floriano, F. J., Morales, J. C., Caballero, J. A., et al. 2015a, A\&A, 577, A128

Alonso-Floriano, F. J., Caballero, J. A., Cortés-Contreras, M., Solano, E., \& Montes, D. 2015b, A\&A, 583, A85

Amado, P. J. Quirrenbach, A., Caballero, J. A., et al. 2013, Highlights of Spanish Astrophysics VII, 842

Ansdell, M., Gaidos, E., Mann, An. W., et al. 2015, ApJ, 798, 41

Baraffe, I., Homeier, D., Allard, F., \& Chabrier, G. 2015, A\&A, 577, A42

Barrado y Navascués, D. 1998, A\&A, 339, 831

Barrado y Navascués, D., Stauffer, J. R., \& Jayawardhana, R. 2004, ApJ, 614, 38

Basri, G., Marcy, G. W., \& Graham, J. R. 1996, ApJ, 458, 600

Bate, M. R. 2012, MNRAS, 419, 3115

Behall, A. L., \& Harrington, R. S. 1976, PASP 88, 204

Béjar, V. J. S., Gauza, B., Caballero, J. A., et al. 2012, 17th Cambridge Workshop on Cool Stars, Stellar Systems, and the Sun, published on-line

Bell, C. P., Mamajek, E. E., \& Naylor, T. 2015, MNRAS, 454, 593

Benavides, R. 2014, Observador de Estrellas Dobles, 12, 21

Benedict, G. F., McArthur, B. E., Franz, O. G., et al. 2000, AJ, 120, 1106

Bergfors, C., Brandner, W., Janson, M., et al. 2010, A\&A, 520, A54

Beuzit, J.-L., Ségransan, D., Forveille, T., et al. 2004, A\&A, 425, 997

Bihain, G., Rebolo, R., Zapatero Osorio, M. R., et al. 2010, A\&A, 519, A93

Bonfils, X., Delfosse, X., Udry, S., et al. 2013, A\&A, 549, A109

Bonnarel, F., Fernique, P., Bienaymé, O., et al. 2000, A\&AS, 143, 33

Bouy, H., Brandnder, W., Martín, E. L., et al. 2003, AJ, 126, 1526

Bowler, B. P., Liu, M. C., Shkolnik, E. L., \& Tamura, M. 2015, ApJS, 216, 7

Burgasser, A. J., Kirkpatrick, J. D., Reid, I. N., et al. 2003, ApJ, 586, 512

Burgasser, A. J., Reid, I. N., Siegler, N., et al. 2007, Protostars and Planets V, 427

Caballero, J. A. 2007, ApJ, 667, 520

Caballero, J. A. 2010, A\&A, 514, A98

Caballero, J. A., Cortés-Contreras, M., López-Santiago, J., et al. 2013, Highlights of Spanish Astrophysics VII, 645 
Charbonneau, D., Berta, Z. K., Irwin, J., et al. 2009, Nature, 462, 891 Christy, J. M. 1978, AJ, 83, 10

Cortés-Contreras, M., Caballero, J. A., Alonso-Floriano, F. J., et al. 2013, Highlights of Spanish Astrophysics VII, 646

Cortés-Contreras, M., Caballero, J. A., \& Montes, D. 2014, The Observatory, 134,348

Cortés-Contreras, M., Béjar, V. J. S., Caballero, J. A. et al. 2015a, Highlights of Spanish Astrophysics VIII, 597

Cortés-Contreras, M., Caballero, J. A., Béjar, V. J. S., et al. 2015b, 18th Cambridge Workshop on Cool Stars, Stellar Systems, and the Sun, 805

Close, L. M., Siegler, N., Freed, M., \& Biller, B. 2003, ApJ, 587, 407

Cruz, K. L., Reid, I. N., Liebert, J., et al. 2003, AJ, 126, 2421

Dawson, P. C., \& De Robertis, M. M. 2005, PASP, 117,

Deacon, N. R., Liu, M. C., Magnier, E. A., et al. 2012, ApJ, 757, 100

Delfosse, X., Forveille, T., Beuzit, J.-L., et al. 1999, A\&A, 344, 897

Desidera, S., Carolo, E., Gratton, R., et al. 2011, A\&A, 533, A90

Dieterich, S. B., Henry, T. J., Golimowski, D. A., et al. 2012, AJ, 144, 64

Dittmann, J. A., Irwin, J. M., Charbonneau, D., \& Berta-Thompson, Z. K. 2014, ApJ, 784, 156

Dommanget, J., \& Nys, O. 2000, A\&A, 363, 991

Duchêne, G., \& Kraus, A. 2013, ARA\&A, 51, 269

Duquennoy, A., \& Mayor, M. 1991, A\&A, 248, 485

Fischer, D. A., \& Marcy, G. W. 1992, ApJ, 396, 178

Freedman, D., \& Diaconis, P. 1981, Probability Theory and Related Field, 57, 453

García-Piquer, A., Morales, J. C., Ribas, I., et al. 2016, A\&A, submitted

Gatewood, G., \& Coban, L. 2009, AJ, 137, 402

Giannuzzi, M. A. 1979, A\&A, 77, 214

Giclas H. L., Burnham Jr. R., \& Thomas N. G. 1971, Lowell proper motion survey Northern Hemisphere (Flagstaff, Arizona: Lowell Observatory)

Gigoyan, K. S., Sinamyan, P. K., Engels, D., \& Mickaelian, A. M. 2010, Astrophysics, 53, 123

Ginski, C., Mugrauer, M., Seeliger, M., et al. 2015, MNRAS, 457, 2173

Gizis, J. E., \& Reid, I. N. 1996, AJ, 111, 365

Gizis, J. E., Reid, I. N., \& Hawley, S. L., et al. 2002, AJ, 123, 3356

Goodwin, S. P., Kroupa, P., Goodman, A., \& Burkert, A. 2007, Protostars and Planets V, 133

Gray, R. O., Corbally, C. J., Garrison, R. F., et al. 2003, AJ, 126, 2048

Gray, R. O., Corbally, C. J., Garrison, R. F., et al. 2006, AJ, 132, 161

Guenther, E. W., \& Tal-Or, L. 2010, A\&A, 521, A83

Guenther, E. W., \& Wuchterl, G. 2003, A\&A, 401, 677

Hartkopf, W. I., \& Mason, B.D. 2011, AJ, 142, 56

Harrington, R. S., \& Dahn C. C. 1980 , AJ, 85, 454

Harrington, R. S., Christy, J. W., \& Strand, K. A. 1981, AJ, 86, 909

Hawley, S. L., Gizis, J. E., \& Reid, I. N. 1996, AJ, 112, 2799

Henry, T. J. 2004, Spectroscopically and Spatially Resolving the Components of the Close Binary Stars, ASP Conf. Ser., 318, 159

Henry, T. J., Jao, W.-C, Subasavage, J. P., et al. 2006, AJ, 132, 2360

Hershey, J. L., \& Taff, L. G. 1998, AJ, 116, 1440

Ireland, M. J., Kraus, A., Martinache, F., et al. 2008, ApJ, 678, 463

Jeffries, R. D., \& Maxted, P. F. L. 2005, Astron. Nachr., 326, 944

Janson, M., Hormuth, F., Bergfors, C., et al. 2012, ApJ, 754, 44

Janson, M., Bergfors, C., Brandner, W., et al. 2014a, ApJ, 789, 102

Janson, M., Bergfors, C., Brandner, W., et al. 2014b, ApJS, 214, 17

Jenkins, L. F. 1952, General catalogue of trigonometric stellar parallaxes (Yale University Observatory, USA)

Jenkins, L. F. 1963, General catalogue of trigonometric stellar parallaxes (Yale University Observatory, USA)

Jenkins, J. S., Ramsey, L. W., Jones, H. R. A., et al. 2009, ApJ, 704, 975

Jódar, E., Pérez-Garrido, A., Díaz-Sánchez, A., et al. 2013, MNRAS, 429, 859

Kirkpatrick, J. D., \& McCarthy, D. W. Jr 1994, AJ, 107, 333

Klutsch, A., Freire Ferrero, R., Guillout, P., et al. 2014, A\&A, 567, A52

Koen, C., Kilkenny, D., van Wyk, F., \& Marang, F. 2010, MNRAS, 403, 1949

Labadie, L., Rebolo, R., Femenía, B., et al. 2010, Proc. SPIE, 7735, E0X

Lafrenière, D., Doyon, R., Marois, C., et al. 2007, ApJ, 670, 1367

Law, N. M. 2006, Ph.D. Thesis, Institute of Astronomy \& Selwyn College, (Cambridge, UK: Cambridge University)

Law, N. M., Hodgkin, S. T., \& Mackay, C. D. 2008, MNRAS, 384, 150

Leinert, C., Henry, T., Glindemann, A., \& McCarthy, D. W. Jr. 1997, A\&A, 325, 159
Lépine, S., Hilton, E. J., Mann, A. W., et al. 2013, AJ, 145, 102 Llamas, M. 2014, MSc Thesis, Universidad Complutense de Madrid, Spain Luhman, K. L. 2012, ARA\&A, 50, 65 Luyten, W. J. 1997, VizieR Online Data Catalog: I/130

Mahadevan, S., Ramsey, L. W., Terrien, R., et al. 2014, Proc. SPIE, 9147, 1

Mamajek, E. E., Bartlett, J. L., Seifahrt, A., et al. 2013, AJ, 146, 154

Martinache, F., Lloyd, J. P., Ireland, M. J., et al. 2007, ApJ, 661, 496

Mason, B. D., Wycoff, G. L., Hartkopf, W. I., Douglass G. G., \& Worley C. E. 2001, AJ, 122, 3466

Mason, B. D., Hartkopf, W. I., \& Friedman, E. A. 2012, AJ, 143, 124

Mason, B. D., Hartkopf, W. I., \& Hurowitz, H. M. 2013, AJ, 146, 56

Montagnier, G., Ségransan, D., Beuzit, J.-L., et al. 2006, A\&A, 460, L19

Montes, D., López-Santiago, J., Gálvez, M. C., et al. 2001, MNRAS, 328, 45

Montes, D., Caballero, J. A., Jeffers, S., et al. 2015, Highlights of Spanish Astrophysics VIII, 605

Montes, D., Caballero, J. A., Gallardo, I., et al. 2016, IAUS, 314, 71

Morrison, J. E., Röser, S., McLean, B., et al. 2001, AJ, 121, 1752

Newton, E. R., Charbonneau, D., Irwin, J., et al. 2014, AJ, 147, 20

Oscoz, A., Rebolo, R., López, R., et al. 2008, Proc. SPIE, 7014, 47

Peña-Ramírez, K., Béjar, V. J. S., \& Zapatero Osorio, M. R. 2016, A\&A, 586, A157

Pettersen, B. R., Evans, D. S., \& Coleman, L. A. 1984, ApJ, 282, 214

Quirrenbach, A., Amado, P. J., Caballero, J. A., et al. 2014, Proc. SPIE, 9147, E1F

Quirrenbach, A., Caballero, J. A., Amado, P. J., et al. 2015, 18th Cambridge Workshop on Cool Stars, Stellar Systems, and the Sun, 18, 897

Raghavan, D., McAlister, H. A., Henry, T. J., et al. 2010, ApJS, 190, 1

Reggiani, M., \& Meyer, M. R. 2011, ApJ, 738, 60

Reggiani, M., \& Meyer, M. R. 2013, A\&A, 553, A124

Reiners, A., \& Basri, G. 2009, ApJ, 705, 1416

Reiners, A., Joshi, N., \& Goldman, B. 2012, AJ, 143, 93

Reid, I. N., \& Cruz, K. L. 2002, AJ, 123, 2806

Reid, I. N., \& Gizis, J. E. 1997, AJ, 113, 2246

Reid, I. N., Hawley, S. L., \& Gizis, J. E. 1995, AJ, 110, 1838

Reid, I. N., Cruz, K. L., Burgasser, A. J., et al. 2004, AJ, 128, 463

Reid, I. N., Cruz, K. L., Burgasser, A. J., \& Liu. M. C. 2008, AJ, 135, 580

Riedel, A. R., Subasavage, J. P., Finch, C. T., et al. 2010, AJ, 140, 897

Riedel, A. R., Finch, C. T., Henry, T. J., et al. 2014, AJ, 147, 85

Riaz, B., Gizis, J. E., \& Harvin, J. 2006, AJ, 132, 866

Röser, S., Demleitner, M., \& Schilbach, E. 2010, AJ, 139, 2440

Rivera, E. J., Lissauer, J. J., Butler, R. P., et al. 2005, ApJ, 634, 625

Sarajedini, A., Bedin, L. R., Chaboyer, B., et al. 2007, AJ, 133, 1658

Scardia, M., Ghiringhelli, D., \& Debehogne, H. 1995, Astron. Nachr., 316, 125

Scardia, M., Prieur, J. L., Pansecchi, L., Argyle, R. W., \& Sala, M. 2011, Astron. Nachr., 332, 508

Scardia, M., Prieur, J. L., Pansecchi, L., et al. 2013, MNRAS, 434, 2803

Scholz, R.-D., Meusinger, H., \& Jahrei $\beta$, H. 2005, A\&A, 442, 211

Shan, Y., Johnson, J. A., \& Morton, T. D. 2015, ApJ, 813, 75

Shkolnik, E. L., Hebb, L., Liu, M. C., et al. 2010, ApJ, 716, 1522

Siegler, N., Close, L. M., Cruz, K. L., et al. 2005, ApJ, 621, 1023

Simon-Díaz, S., Caballero, J. A., Lorenzo, J., et al. 2015, ApJ, 799, 169

Soderblom, D. R., \& Mayor, M. 1993, AJ, 105, 226

Strand K. A. A. 1977, AJ, 82, 9

Subasavage, J. P., Jao, W.-C., Henry, T. J., et al. 2009, AJ, 137, 4547

Tamura, M., Suto, H., Nishikawa, J., et al. 2012, Proc. SPIE, 8446, 1

Thorel, J. C., Thorel, Y., \& Verhas, P. 2011, Observations \& Travaux, 78, 20

Tody, D. 1986, Proc. SPIE, 627, 733

Tokovinin, A. 2008, MNRAS, 389, 925

Tokovinin, A. 2011, AJ, 141, 52

Tokovinin, A., Mason, B. D., Hartkopf, W. I., et al. 2015, AJ, 150, 50

van Altena, W. F., Lee, J. T., \& Hoffleit, D. 1995, General catalogue of trigonometric stellar parallaxes (Yale University Observatory, USA) van Leeuwen, F. 2007, A\&A, 474, 653

Wang, J., Xie, J.-W., Barclay, T., \& Fischer, D. A. 2014a, ApJ, 783, 4

Wang, J., Fischer, D. A., Xie, J.-W., \& Ciardi, D. R. 2014b, ApJ 791, 111

Wang, J., Fischer, D. A., Horch, E. P., \& Xie, J.-W 2015a, ApJ, 806, 248

Wang, J., Fischer, D. A., Xie, J.-W \& Ciardi, D. R. 2015b, ApJ, 813, 130

Weinberger, A. J., Boss, A. P., Keiser, S. A., et al. 2016, AJ, 152, 24

Ward-Duong, K., Patience, J., De Rosa, R. J., et al. 2015, MNRAS, 449, 2618

Zuckerman, B., \& Song, I. 2014, ARA\&A, 42, 685 